\title{
Burnett Order Stress and Spatially-Dependent Boundary Conditions for the Lattice Boltzmann Method
}

\author{
Timothy Reis* \\ School of Computing and Mathematical Sciences, University of Greenwich, \\ London SE10 9LS, UK.
}

Received 7 September 2018; Accepted (in revised version) 11 February 2019

\begin{abstract}
Stress boundary conditions for the lattice Boltzmann equation that are consistent to Burnett order are proposed and imposed using a moment-based method. The accuracy of the method with complicated spatially-dependent boundary conditions for stress and velocity is investigated using the regularized lid-driven cavity flow. The complete set of boundary conditions, which involve gradients evaluated at the boundaries, are implemented locally. A recently-derived collision operator with modified equilibria and velocity-dependent collision rates to reduce the defect in Galilean invariance is also investigated. Numerical results are in excellent agreement with existing benchmark data and exhibit second-order convergence. The lattice Boltzmann stress field is studied and shown to depart significantly from the Newtonian viscous stress when the ratio of Mach to Reynolds numbers is not negligibly small.
\end{abstract}

AMS subject classifications: 65Z05, 76A05, 76D05

Key words: Lattice Boltzmann method, moment based boundary conditions, Burnett stress, regularized cavity.

\section{Introduction}

Since its initial developments [1-3], the lattice Boltzmann method (LBM) has become an established branch of computational fluid dynamics (CFD). Its foundations can appear to be conceptually different from traditional methods of CFD: rather than discretising continuum mass, momentum, and possibly constitutive equations directly, the LBM is derived from a velocity-space truncation of Boltzmann's equation for gases [4]. This kinetic formulation yields a linear, constant coefficient hyperbolic system of equations in which all nonlinearities are confined to algebraic source terms. The linear differential operators are discretised exactly by integrating along their characteristics and the governing equations of motion are recovered by seeking slowly varying solutions to the kinetic

${ }^{*}$ Corresponding author. Email address: T.Reis@greenwich.ac.uk (T. Reis) 
equation. The locality of the lattice Boltzmann algorithm gives it the opportunity to exploit massively parallel modern computer architectures, including graphics processing units (GPUs), leading to very fast computations [5].

The lattice Boltzmann equation converges with second-order accuracy in grid spacing to the solution of the discrete Boltzmann equation, which at fixed Mach and Reynolds numbers differ from the (weakly) compressible Navier-Stokes equations only by finite Mach number artefacts and $\mathcal{O}\left(K n^{2}\right)$ contributions to the stress, where $K n$ is the Knudsen number [6-8]. By von-Karman's relation, $K n \propto M a / R e$. The finite Mach number errors in standard lattice Boltzmann models are of order $\mathcal{O}\left(M a^{3}\right)$. They appear in the stress tensor and break Galilean invariance. They arise because the discrete Maxwellian equilibria are truncated at second-order in velocity and cannot be removed completely on the standard nearest neighbour integer lattices that are commonly in use (e.g D2Q9, D3Q15, D3Q19, or D3Q27 lattices). Hazi and Kavran [9] proposed adjusting the third order moment to restore Galilean invariance in axis-aligned shear flows but defects still existed in the diagonal components of the momentum flux tensor. Building on this, Dellar [10] showed that these defects can be eliminated when the LBM density is constant and reduced to $\mathcal{O}\left(M a^{5}\right)$ otherwise if a collision operator with velocity-dependent relaxation times is used. A similar approach was proposed by Geier et al. [11] to reduce the defects under diffusive scaling by adjusting the equilibria in the cumulant lattice Boltzmann method.

Although usually neglected, the deviatoric stress that is naturally embedded in the LBM moment PDE system is governed by non-Newtonian constitutive equation that includes "Burnett order" contributions, as shown by Dellar [12]. Thus, computations of the stress with the LBM with large relaxation times can yield physically meaningful results that do not agree with the Navier-Stokes equations. This has implications for lattice Boltzmann methods for rarefied flow, the LBM at low Reynolds numbers, and the calculation of forces on bodies using the LBM (since the non-equilibrium contribution to the moment flux $\left.\Pi_{\alpha \beta}^{(1)}=-\mu\left(\partial_{\alpha} u_{\beta}+\partial_{\beta} u_{\alpha}\right)+\mathcal{O}\left(\tau^{2}\right)\right)$ and, as discussed in detail here, the implementation of boundary conditions. Imposing stress boundary conditions that are local, secondorder accurate, and consistent with the moments of the truncated Boltzmann PDE is not easily achieved with most lattice Boltzmann implementations. Furthermore, the stress at a boundary may depend on the velocity in a non-trivial way and even constraints on the velocity field can be difficult to implement precisely with lattice Boltzmann when $\mathbf{u}_{\text {wall }}=\mathbf{u}_{\text {wall }}(\mathbf{x})$ is not constant.

At first, imposing boundary conditions on the lattice Boltzmann algorithm appears straightforward: one must supply values for the distributions $f_{i}$ (see equation 2.1) on "incoming" characteristics pointing into the domain. The popular D2Q9 lattice (Fig. 2) has three unknown incoming distributions at boundaries aligned with grid points and lattice Boltzmann boundary conditions specify the values of these distribution functions in terms of those that are known. Incoming distributions at boundary nodes can be determined by a simple reversal in the particles' velocity; an approach known as "bounceback". Some steady state solutions of the linearised (continuous) Boltzmann equation 
with bounce-back boundary conditions were found by Cercignani [13]. Ladd [14] showed that the lattice Boltzmann method with bounce-back and momentum exchange boundary conditions can recover exact solutions to Couette flow (a linear velocity profile) when the boundary is placed precisely half-way between layers of grid points. His simulations of force-driven Poiseuille flow, with bounce-back boundary conditions, however, were subject to error. It is now well-known that this error is due to an unphysical "numerical slip" at solid boundaries that is second-order in grid spacing, $\Delta x$, provided the boundary is placed correctly between grid points [7,15].

The slip error can be removed using a multiple relaxation time (MRT) or a two relaxation time (TRT) model with a very specific relationship between the relaxation times of the momentum flux (which defines the viscosity) and the third moment of the lattice distribution functions: the numerical slip is eliminated if the product of these two relaxation times, often called the "magic parameter" $\Lambda$, is set to be $3 / 16$ in so-called lattice units [15]. That is, the boundary condition on the third moment is tuned until the tangential velocity vanishes at the required location. The magic parameter $\Lambda$ also affects the effective stencil for the flux of the non-conserved moments and thus influences the stability and accuracy of the entire algorithm [16-19]. In particular, $\Lambda=1 / 4$ removes the recurrence in higher moments, and so is sometimes considered to be the most stable choice of relaxation times [16,17]. The choice $\Lambda=1 / 6$ removes a fourth-order spatial error and $\Lambda=1 / 12$ a third order spatial error $[19,20]$ but none of these numerically favourable options eliminate the numerical artefact due to bounce-back boundary conditions. In addition, it is not always easy to impose hydrodynamic boundary conditions other than no-slip for stationary walls with the bounce-back methods. But it must be noted that, despite some known errors, the bounce-back method is often the most useful way of implementing boundary conditions for the LBM in complex geometries: as well as its flexibility in irregular domains, in comparison with other methods it is numerically stable at quite high Re numbers even when used with the BGK collision operator, and the spurious slip is small when $\tau$ is very small.

Maxwell's diffuse reflection boundary condition [21] involves the incoming Maxwellian distribution function with the overall amplitude set to zero to ensure zero mass flux through the wall. The wall velocity is specified in the Maxwellian, allowing the effects of moving walls to be incorporated with relative ease, while the wall density is found from the zero mass flux condition. A discretisation of Maxwell's diffuse reflection boundary condition to a finite set of particle velocities was first proposed by Broadwell [22] and studied further by Gatignol [23]. This approach has since been adopted by Ansumali and Karlin for the lattice Boltzmann equation [24] and studied further by Kim et al. [25] and Brookes et al. [26]. In steady unidirectional flows, the on-node D2Q9 implementation of Maxwell-Broadwell boundary conditions with a unit accommodation coefficient (diffuse reflection) is equivalent to setting the incoming distribution functions to their equilibrium values [27]. The numerical slip velocity persists [28] and is one order in grid spacing, $\Delta x$, larger than in bounce-back [7]. In fact, all lattice Boltzmann models which have distributions of particles travelling tangential to a boundary will include a 
numerical slip error if the applied boundary conditions do not explicitly consider these "grazing" molecules [23].

Most LBM implementations of boundary conditions are formulated in terms of the distribution functions, $f_{i}$. However, hydrodynamics are recovered from moments of the $f_{i}$. The lattice Boltzmann equation has a finite set of particle velocity distribution functions which can be mapped to a moment basis by a linear, invertible, transformation. Thus an alternative method for implementing boundary conditions would be to impose conditions on the hydrodynamic moments at a boundary and then translate these into conditions for the unknown variables. This needs to be done in a way that uniquely determines the incoming $f_{i}$ (see Section 5 and Table 1). The spirit of this method was first proposed by Noble et al. [29] who imposed constraints on the two fluid velocity components, $\mathbf{u}=0$, to determine the two incoming distributions at an axis-aligned planar boundary on the hexagonal FHP lattice [30]. The D2Q9 lattice is more complicated. It has three incoming distributions at boundaries aligned with grid points and thus requires three moment constraints. This was recognised by Wagner and Yeomans [31] who used a similar methodology to impose continuity of the normal velocity and tangential component of the pressure tensor for Lees-Edwards sliding periodic boundary conditions for shear flow. Bennett [32] generalised the approach of Noble et al. [29] with the "momentbased method". The moment-based method may be considered as a systematic study of which moment conditions are compatible with uniquely determining incoming distribution functions. At solid boundaries aligned with grid points, for example, it usually imposes conditions on each component of the momentum and an additional boundary condition on the tangential component of the momentum flux (i.e the stress); the only remaining independent hydrodynamic moment for the D2Q9 velocity set (see Table 1 in Section 5). This approach, like all other on-node boundary conditions, is not easily extended to complicated geometries where boundaries are not aligned with grid points. It does, however, provide a general methodology for imposing hydrodynamic conditions (on velocity, pressure, or stress) locally and precisely at grid points and has been shown to be very accurate [33]. The moment-based method has already been employed to implement diffusive slip in binary gases [34], Navier-Maxwell slip [35,36], contact angles in multiphase flows [37], and Dirichlet and von-Neumam conditions for natural convection [38].

Previous applications of the moment method determine the tangential momentum flux at a boundary by imposing $\partial_{x} u_{x}=0$. Moreover, it was always implicitly assumed that the deviatoric stress, $T$, is given by Newtonian constitutive equation [32-35,37-39]. A closer inspection shows that the stress obtained from the D2Q9 discrete velocity Boltzmann equation includes a non-zero Burnett contribution at second-order in relaxation time that reassembles a non-objective viscoelastic constitutive equation [12]. Thus, the Navier-Stokes stress boundary condition is inconsistent with the underlying partial differential equation and a correct, more sophisticated, set of constraints is sought. This becomes yet more complicated with a boundary moving with a spatially-dependent velocity, as is the case with the regularised lid-driven cavity flow. 

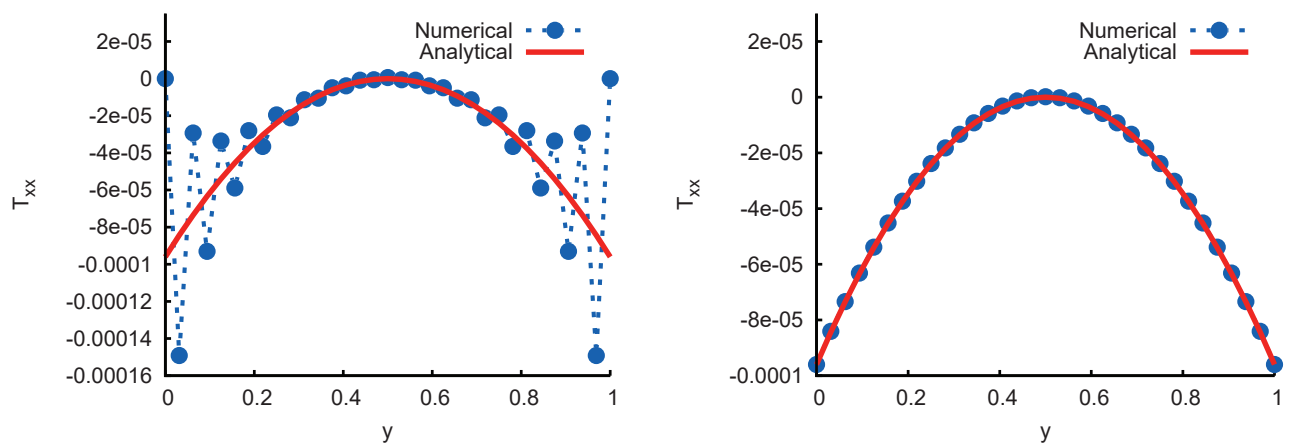

Figure 1: Plot of the tangential stress in planar channel flow using the LBM with moment-based boundary conditions ("Numerical") compared with the solution from the discrete Boltzmann equation ("Analytical"). The plot on the left uses the original Navier-Stokes stress condition and the plot on the right the Burnett stress condition.

To demonstrate and highlight this inconsistency we consider the indicative case of steady planar channel flow with no-slip walls. The velocity field is one-dimensional, $\mathbf{u}=\left(u_{x}(y), 0\right)$, so the tangential component of the stress in the Navier-Stokes equations vanishes, $T_{x x}=0$. However, the solution computed by the lattice Boltzmann equation is somewhat different, as shown in Fig. 1. Here the Reynolds number in $R e=100$ and the parabolic profile is indicative of behaviour beyond the Navier-Stokes level of description. The general quadratic solution comes from the lattice Boltzmann equation, and not its boundary conditions. In the plot on the left of Fig. 1 the original moment-based method with the "Navier-Stokes" stress boundary condition has been used. The spurious oscillations emanating from the walls are striking. We argue that these arise from imposing a boundary condition for the Navier-Stokes stress, rather than for the stress embedded in the discrete Boltzmann equation. More specifically, the solution of the tangential stress from the discrete Boltzmann equation in this flow is $T_{x x}=-2 \mu \tau\left(u_{x}^{\prime}\right)^{2}$ (c.f. Section 4.1), where $\mu$ is the dynamic viscosity, $\tau$ is the BGK relaxation time, and the prime denotes differentiation with respect to the normal coordinate $y$. This stress does not vanish at solid walls but the original moment-based boundary conditions sets $T_{x x}=0$. This mismatch causes oscillations in the numerical solution at the walls that can infect the entire flow domain, possibly generating numerical instabilities. By imposing a consistent condition for the stress (see Section 5) we can remove the spurious behaviour and compute very accurate solutions to the tangential stress. It should be noted that the moment-based method with the Navier-Stokes stress conditions gives the exact solution for the velocity in Poiseuille flow on just three grid points.

Lid-driven cavity flow has been used many times to assess lattice Boltzmann models. Hou et al. [40] provided the first detailed numerical evaluation of LBE computations of cavity flow but more recent developments have allowed for a significant improvement in accuracy [41]. Gorban and Packwood [42] and Brownlee et al. [43] used the classical liddriven cavity flow to study the stability of lattice Boltzmann algorithms and concluded 
that multiple relaxation time models and filtering techniques are superior to BGK and entropic collision models. Mohammed and Reis [39] used the cavity flow to assess the moment-based method with simple boundary conditions and although they reported very accurate results in agreement with the most sophisticated numerical methods when using an MRT collision operator they also found that the method becomes unstable at higher Reynolds numbers on coarse meshes when used with the BGK collision operator (as is this case with other on-node methods - they are less stable than bounce back when used with BGK). The classic lid-driven cavity flow has stress singularities in the upper corners where the moving horizontal lid meets stationary vertical walls. In was suggested in [39] that this may be the cause of instabilities of the moment-based boundary conditions at high $R e$ on coarse meshes. To circumvent the singularity we simulate here the so-called "regularized" cavity [44], where the velocity of the lid (north horizontal boundary) in box $0 \leq x, y \leq 1$ is a smooth function of the horizontal coordinate $x$ :

$$
\left.\mathbf{u}\right|_{\text {lid }}=\left(u_{x}(x, 1), u_{y}(x, 1)\right)=\left(16 x^{2}(1-x)^{2}, 0\right), \quad 0 \leq x \leq 1,
$$

rather than a constant. All boundaries are no-slip and $\mathbf{u}=(0,0)$ at all other boundaries. The maximum velocity of the lid is $u_{x}(1 / 2,1)=1$. Like the simpler and more common constant velocity case, characteristic features of this flow include the development of vortex regions in the cavity: for small to moderate Reynolds numbers a primary vortex develops near the centre of the domain with two weaker ones in the vicinity of the bottom corners; for larger Reynolds numbers a further vortex develops in the upper left-hand corner.

The velocity condition (1.1) influences the stress field since it has non-vanishing gradients. This is important for moment-based methods that attempt to impose hydrodynamic constraints directly (see Section 5). Moveover, this adds an extra layer of complexity when one realises that the constitutive equation for stress is non-Newtonian. More specifically, it will be shown in Section 5 that the tangential stress on the boundary that needs to be implement in the lattice Boltzmann algorithm is

$$
T_{x x}=\frac{2 \mu\left(\partial_{x} u_{x}-\tau u_{x} \partial_{x x} u_{x}\right)-2 \frac{T_{x y}^{2}}{\Pi_{y y}}}{1+2 \tau \partial_{x} u_{x}}+\mathcal{O}\left(M a^{3}\right),
$$

where $\mu$ is the dynamic viscosity of the fluid, $\tau$ is the stress relaxation time, $T_{x y}$ is the off-diagonal component of the deviatoric stress, and $\Pi_{y y}$ is the normal component of the momentum flux. It is clear that it is non-Newtonian, for otherwise $T_{x x}=2 \mu \partial_{x} u_{x}$ (and for a Newtonian fluid with a homogenous velocity boundary conditions $T_{x x}=0$ ) and not easily implemented with methods formulated in terms of the distribution functions. Despite their complexity, these quite general stress and velocity conditions can be imposed simply and locally at grid points with the moment-based methods discussed in this article. The method developed here is validated against data from spectral simulations and used to examine the LBM stress field in low Reynolds number flows. Furthermore, an LBM without cubic defects in Galilean invariance which is shown to reduce the Mach number error in Eq. (1.2) to $\mathcal{O}\left(M a^{5}\right)$ is discussed and used for additional assessment. 


\section{The discrete Boltzmann equation}

The discrete Boltzmann partial differential equation

$$
\frac{\partial f_{i}}{\partial t}+\boldsymbol{\xi}_{i} \cdot \nabla f_{i}=-\frac{1}{\tau}\left(f_{i}-f_{i}^{(0)}\right)
$$

describes the evolution of a finite set of distribution functions $f_{i}$ corresponding to populations of particles moving with a finite set of discrete particle velocities $\left\{\boldsymbol{\xi}_{i}\right\}[4,45]$. The left hand side of Eq. (2.1) describes the propagation of $f_{i}$ with velocity $\xi_{i}$. The right hand side of Eq. (2.1) describes the net effect of particle interactions, which is to relax the distributions to their local equilibria $f_{i}^{(0)}$ with a single relaxation time $\tau$ (the BGK operator [46]). We focus on the D2Q9 lattice [2] shown in Fig. 2 but the methodology presented here is applicable to other discrete velocity sets.

The equilibria $f_{i}^{(0)}$ are functions of the fluid density $\rho$ and velocity $\mathbf{u}$, as defined in Eq. (2.4) below, and can be derived from a small Mach number $M a=|\mathbf{u}| / c_{s}\left(c_{s}=c / \sqrt{3}\right.$ for D2Q9 when $c \gg|\mathbf{u}|$ is the particle speed) expansion of the discretised (in particle velocity space) Maxwell-Boltzmann distribution. Up to second-order in $M a$ the equilibrium functions are $[2,4]$,

$$
f_{i}^{(0)}=w_{i} \rho\left(1+3 \xi_{i} \cdot \mathbf{u}+\frac{9}{2}\left(\boldsymbol{\xi}_{i} \cdot \mathbf{u}\right)^{2}-\frac{3}{2}|\mathbf{u}|^{2}\right),
$$

where the D2Q9 weights are given by

$$
w_{i}= \begin{cases}4 / 9, & i=0, \\ 1 / 9, & i=1,2,3,4, \\ 1 / 36, & i=5,6,7,8 .\end{cases}
$$

Macroscopic quantities are defined as the moments of $f_{i}$. The set of 9 particle velocities permits 9 independent moments. Six of these correspond to the hydrodynamic quantities of density, momentum, and momentum flux:

$$
\rho=\sum_{i} f_{i} ; \quad \rho \mathbf{u}=\sum_{i} f_{i} \xi_{i} ; \quad \Pi=\sum_{i} f_{i} \xi_{i} \xi_{i} .
$$

Taking moments of the discrete Boltzmann equation (2.1) yields the evolution equations

$$
\begin{aligned}
& \partial_{t} \rho+\nabla \cdot(\rho \mathbf{u})=0, \\
& \partial_{t}(\rho \mathbf{u})+\nabla \cdot \boldsymbol{\Pi}=0, \\
& \partial_{t} \boldsymbol{\Pi}+\nabla \cdot \boldsymbol{Q}=-\frac{1}{\tau}\left(\boldsymbol{\Pi}-\boldsymbol{\Pi}^{(0)}\right),
\end{aligned}
$$

where $Q=\sum_{i} f_{i} \xi_{i} \xi_{i} \xi_{i}$. The right hand sides of Eqs. (2.5a) and (2.5b) vanish because mass and momentum are conserved by collisions (moments of Eq. (2.2) give $\sum_{i} f_{i}^{(0)}=\rho$ and $\left.\sum_{i} \xi f_{i}^{(0)}=\rho \mathbf{u}\right)$. The momentum flux $\Pi$ relaxes towards its equilibrium $\Pi^{(0)}$ on the collisional timescale $\tau$, as shown by Eq. (2.5c). 


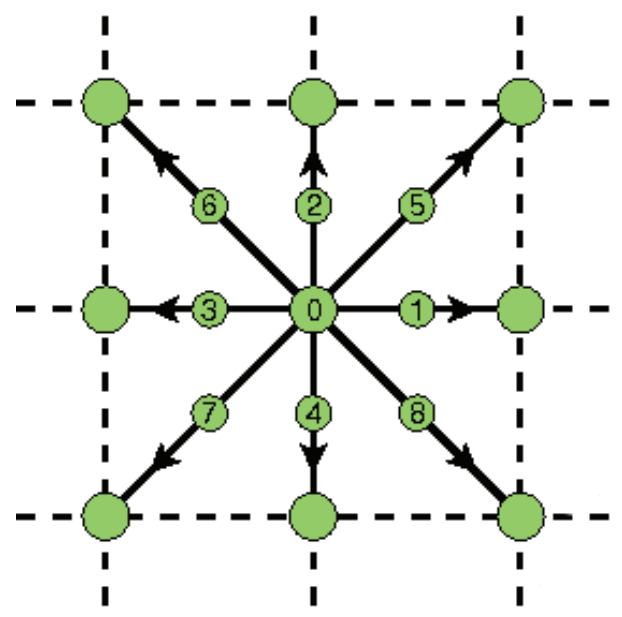

Figure 2: The nine particle propagation velocities $\xi_{0}, \ldots, \xi_{8}$ in the D2Q9 integer lattice.

\subsection{Classical recovery of fluid equations}

We seek solutions to the discrete Boltzmann equation (2.1) that vary slowly over timescales much longer than the collision time $\tau$. That is, we expand the non-conserved moments and the time derivative as a series in the collision time $\tau$ :

$$
\Pi=\Pi^{(0)}+\tau \Pi^{(1)}+\cdots, \quad Q=Q^{(0)}+\tau Q^{(1)}+\cdots, \quad \partial_{t}=\partial_{t_{0}}+\tau \partial_{t_{1}}+\cdots .
$$

The expansion of the time derivative is necessary to suppress secular terms that would otherwise disorder the expansion on long timescales $t=\mathcal{O}(1 / \tau)[47,48]$. Substituting the above into the moment system (2.5a)-(2.5c) and neglecting terms of $\mathcal{O}\left(\tau^{2}\right)$ yields, after some algebra,

$$
\begin{aligned}
& \partial_{t} \rho+\nabla \cdot(\rho \mathbf{u})=0, \\
& \partial_{t}(\rho \mathbf{u})+\nabla \cdot\left(\boldsymbol{\Pi}^{(0)}+\tau \boldsymbol{\Pi}^{(1)}\right)=0
\end{aligned}
$$

where

$$
\boldsymbol{\Pi}^{(0)}=\frac{1}{3} \rho \mathbf{I}+\rho \mathbf{u} \mathbf{u}, \quad \boldsymbol{\Pi}^{(1)}=-\frac{1}{3} \rho\left(\nabla \mathbf{u}+(\nabla \mathbf{u})^{\top}\right)+\nabla \cdot(\rho \mathbf{u} \mathbf{u u}) .
$$

The last term in $\Pi^{(1)}$ is an error term of $\mathcal{O}\left(M a^{3}\right)$ which breaks Galilean invariance [49]. A method for restoring Galilean invariance completely for incompressible flows, and reducing the defect to $\mathcal{O}\left(M a^{5}\right)$ otherwise, was recently proposed by Dellar [10]. This will be discussed further in Section 3.2. A similar approach for restoring or improving to the same order Galilean invariance was developed under the diffusive scaling by Geier $e t$ al. [11]. 


\section{Lattice Boltzmann implementation}

A numerical algorithm for solving the discrete Boltzmann equation can be obtained by first integrating (2.1) along a characteristic for time $\Delta t$ :

$$
f_{i}\left(\mathbf{x}+\boldsymbol{\zeta}_{i} \Delta t, t+\Delta t\right)-f_{i}(\mathbf{x}, t)=\int_{0}^{\Delta t} \mathcal{C}_{i}\left(\mathbf{x}+\boldsymbol{\zeta}_{i} s, t+s\right) d s,
$$

where $\mathcal{C}_{i}$ represents the collision operator on the right-hand side of (2.1). The left hand side of (3.1) is exact, and the integral on the right-hand side can be approximated using the trapezoidal rule:

$$
f_{i}\left(\mathbf{x}+\xi_{i} \Delta t, t+\Delta t\right)-f_{i}(\mathbf{x}, t)=\frac{\Delta t}{2}\left(\mathcal{C}_{i}\left(\mathbf{x}+\xi_{i} \Delta t, t+\Delta t\right)+\mathcal{C}_{i}(\mathbf{x}, t)\right)+\mathcal{O}\left(\Delta t^{3}\right)
$$

This is a second-order accurate but implicit system of algebraic equations. Following He et al. [50] we perform the change of variables

$$
\bar{f}_{i}(\mathbf{x}, t)=f_{i}(\mathbf{x}, t)+\frac{\Delta t}{2 \tau}\left(f_{i}(\mathbf{x}, t)-f_{i}^{(0)}(\mathbf{x}, t)\right) .
$$

The previous implicit scheme (3.2) can now be rearranged into an explicit algorithm for the $\bar{f}_{i}$ at the new timestep:

$$
\bar{f}_{i}\left(\mathbf{x}+\boldsymbol{\zeta}_{i}, t+\Delta t\right)=\bar{f}_{i}(\mathbf{x}, t)-\frac{\Delta t}{\tau+\Delta t / 2}\left(\bar{f}_{i}(\mathbf{x}, t)-f_{i}^{(0)}(\mathbf{x}, t)\right) .
$$

Thus we discard the $f_{i}$ and evolve instead the $\bar{f}_{i}$ according to (3.4). The grid spacing and time step are related to each other through the discrete particle velocity $c=\Delta x / \Delta t \gg 1$.

Density and momentum are conserved by collisions, and so can be obtained directly from moments of the $\bar{f}_{i}$ :

$$
\rho=\sum_{i} f_{i}=\sum_{i} \bar{f}_{i}, \quad \rho \mathbf{u}=\sum_{i} f_{i} \boldsymbol{\xi}_{i}=\sum_{i} \bar{f}_{i} \boldsymbol{\xi}_{i}
$$

Non-conserved moments, such as the momentum flux $\Pi$ (which includes the stress), are found by taking moments of the transformation equation (3.3), which gives

$$
\Pi=\frac{2 \tau \bar{\Pi}+\Delta t \Pi^{(0)}}{2 \tau+\Delta t}
$$

where $\overline{\boldsymbol{\Pi}}=\sum_{i} \bar{f}_{i} \xi_{i} \xi_{i}$. 


\subsection{Multiple relaxation times}

The D2Q9 lattice Boltzmann equation has 9 independent moments but only 6 of these are needed to recover the Eqs. (2.7), (2.8), and (2.9). The three additional "ghost" moments do not directly contribute to these equations but they can have a profound effect on the numerical stability of the lattice Boltzmann algorithm. To simulate flows at high Reynolds numbers without excessive resolution, one must generally over-relax the velocity distribution functions, $\bar{f}_{i}$, rather than relaxing them to equilibrium monotonically [51]. This causes the non-equilibrium part of $\bar{f}_{i}$ to oscillate from timestep to timestep; potentially jeopardising numerical stability. Multiple relaxation time (MRT) models are usually constructed to over-relax the hydrodynamic moments only, while damping oscillations in the ghost (or "kinetic") moments. For a detailed discussion of the theory of, and motivation for, MRT models, we refer the reader to [3,14,42,43,51,52].

Following Dellar [51], we construct an orthogonal basis in $\mathbb{R}^{9}$ with respect to the weighted inner product with weights $w_{i}$ by defining the non-hydrodynamic, or "ghost", moments

$$
\mathcal{J}=\sum_{i=1}^{8} h_{i} f_{i} \xi_{i}, \quad \mathcal{N}=\sum_{i=1}^{8} h_{i} f_{i}
$$

where $h_{i}=(1,-2,-2,-2,-2,4,4,4,4)^{T}$. The moments $\mathcal{J}_{x}, \mathcal{J}_{y}$ and $\mathcal{N}$ are related to $Q_{x x y}$, $Q_{x y y}$, and $R_{x x y y}$ as follows:

$$
\begin{aligned}
& Q_{x x y}=\frac{1}{3} \rho u_{y}+\frac{1}{6} \mathcal{J}_{y}, \quad Q_{x y y}=\frac{1}{3} \rho u_{x}+\frac{1}{6} \mathcal{J}_{x}, \\
& R_{x x y y}=-\frac{1}{9} \rho+\frac{1}{3} \Pi_{x x}+\frac{1}{3} \Pi_{y y}+\frac{1}{9} \mathcal{N} .
\end{aligned}
$$

The velocity distribution function $\bar{f}_{i}$ can now be written as

$$
\bar{f}_{i}=w_{i}\left(\rho+3(\rho \mathbf{u}) \cdot \boldsymbol{\xi}_{i}+\frac{9}{2}\left(\overline{\mathbf{\Pi}}-\frac{\rho}{3} \mathbf{I}\right):\left(\xi_{i} \boldsymbol{\xi}_{i}-\frac{1}{3} \mathbf{I}\right)\right)+w_{i} h_{i}\left(\frac{1}{4} \overline{\mathcal{N}}+\frac{3}{8} \xi_{i} \cdot \overline{\mathcal{J}}\right)
$$

where the "barred" variables are moments of $\bar{f}_{i}$. The equilibrium ghost moments are $\overline{\mathcal{N}}^{(0)}=\overline{\mathcal{J}}_{\alpha}^{(0)}=0$. One now performs collisions directly upon the non-conserved moments $\bar{\Pi}, \overline{\mathcal{J}}$, and $\overline{\mathcal{N}}$ (each potentially relaxing according to its own collisional timescale) and then reconstructs the post collision distribution function according to Eq. (3.10). A common MRT model ensures that the non-hydrodynamic moments $\overline{\mathcal{J}}$ and $\overline{\mathcal{N}}$ decay instantaneously to the equilibria (which are zero) after collisions (so that their relaxation times are $1 / 2$ in lattice units). This approach was proposed initially by Ladd [14] and has recently been referred to as the "regularized" lattice Boltzmann equation [53].

Two-relaxation time (TRT) models relax odd and even order moments at different rates. That is, $\Pi$ and $\mathcal{N}$ relax to their equilibria with a relaxation time $\tau$ determined by the fluid velocity, and $\mathcal{J}$ relaxes to its equilibrium according to a different relaxation 
time, $\tau_{J}$, judiciously chosen to optimise numerical stability. We use two values of the magic parameter $\Lambda=\tau \tau$ J. The choice $\Lambda=1 / 4$ truncates the recurrence relation for the non-conserved moments, and has been shown to offer favourable stability properties $[16,17,54]$, while $\Lambda=1 / 6$ removes a fourth-order spatial error $[19,20]$.

\subsection{Cubic correction to the stress tensor}

The third-order moment $\boldsymbol{Q}^{(0)}$ of the (continuous) Maxwell-Boltzmann equilibrium function has terms of third order in Mach number [46]. These are missing in the third moment of the lattice Boltzmann quadratic equilibria (2.2) and their absence results in the $\tau \nabla \cdot(\rho \mathbf{u u u})$ error term in the viscous stress tensor $\Pi^{(1)}$ in Eq. (2.9) that breaks Galilean invariance. The $\mathrm{D} 2 \mathrm{Q} 9$ lattice does not have enough degrees of freedom to specify all components of $\mathbf{Q}^{(0)}$ independently of lower order moments.

The off-diagonal components of $\mathbf{Q}^{(0)}$ can be corrected on the D2Q9 lattice and set to [9]

$$
Q_{x x y}^{(0)}=\frac{1}{3} \rho u_{y}+\rho u_{x}^{2} u_{y}, \quad Q_{x y y}^{(0)}=\frac{1}{3} \rho u_{x}+\rho u_{x} u_{y}^{2} .
$$

Thus, from Eqs. (3.8) and (3.9),

$$
\begin{aligned}
& \mathcal{J}_{x}^{(0)}=6 \rho u_{x} u_{y}^{2} \\
& \mathcal{J}_{y}^{(0)}=6 \rho u_{x}^{2} u_{y} .
\end{aligned}
$$

This is sufficient to correct the error in $\Pi_{x y}^{(1)}$ but not in $\Pi_{x x}^{(1)}$ or $\Pi_{y y}^{(1)}$. However, it can be shown that

$$
\begin{aligned}
\tau \Pi_{x x}^{(1)} & =-\frac{2}{3} \rho \tau \partial_{x} u_{x}+\tau \partial_{x}\left(\rho u_{x}^{3}\right) \\
& =-\frac{2}{3} \rho \tau \partial_{x} u_{x}+3 \tau \rho u_{x}^{2} \partial_{x} u_{x}+\tau u_{x}^{3} \partial_{x} \rho \\
& =-\frac{2}{3} \rho \tau\left(1-\frac{9 u_{x}^{2}}{2}\right) \partial_{x} u_{x}+\tau u_{x}^{3} \partial_{x} \rho
\end{aligned}
$$

A similar expression can be found for $\tau \Pi_{y y}^{(1)}$. Following Dellar [10], the lattice Boltzmann collision operator can be adjusted to apply a relaxation time $\tau_{x x}=\tau\left[1-9 u_{x}^{2} / 2\right]^{-1}$ to $\Pi_{x x}$. If we replace $\tau$ in the last line of Eq. (3.13) with $\tau_{x x}, \tau \Pi_{x x}^{(1)}$ becomes

$$
\tau \Pi_{x x}^{(1)}=-\frac{2}{3} \rho \tau \partial_{x} u_{x}+\tau\left(\frac{2}{2-9 u_{x}^{2}}\right)^{-1} u_{x}^{3} \partial_{x} \rho
$$

The second term in Eq. (3.14) is $\mathcal{O}\left(M a^{5}\right)$, thus reducing the non-Galilean invariant error by two orders in Mach number. Likewise, the error in $\tau \Pi_{y y}^{(1)}$ can be reduced by $\mathcal{O}\left(M a^{2}\right)$ by taking its collision time to be $\tau_{y y}=\tau\left[1-9 u_{y}^{2} / 2\right]^{-1}$. 


\subsection{Implementation of the collision step}

We implement the cubic correction model with multiple relaxation times as follows. The non-conserved moments relax to their equilibria according to

$$
\begin{aligned}
& \bar{\Pi}_{x y}^{\prime}=\bar{\Pi}_{x y}-\frac{\Delta t}{\tau+\Delta t / 2}\left(\bar{\Pi}_{x y}-\Pi_{x y}^{(0)}\right), \\
& \bar{\Pi}_{x x}^{\prime}=\bar{\Pi}_{x x}-\frac{\Delta t}{\tau_{x x}+\Delta t / 2}\left(\bar{\Pi}_{x x}-\Pi_{x x}^{(0)}\right), \\
& \bar{\Pi}_{y y}^{\prime}=\bar{\Pi}_{y y}-\frac{\Delta t}{\tau_{y y}+\Delta t / 2}\left(\bar{\Pi}_{y y}-\Pi_{y y}^{(0)}\right), \\
& \overline{\mathcal{J}}^{\prime}=\overline{\mathcal{J}}-\frac{\Delta t}{\tau_{J}+\Delta t / 2}\left(\overline{\mathcal{J}}-\mathcal{J}^{(0)}\right), \\
& \overline{\mathcal{N}}^{\prime}=\overline{\mathcal{N}}-\frac{\Delta t}{\tau_{N}+\Delta t / 2}\left(\overline{\mathcal{N}}-\mathcal{N}^{(0)}\right),
\end{aligned}
$$

where the primes denote post-collisional quantities and $\mathcal{N}^{(0)}=0$. The collision algorithm is then:

1. compute the moments of $\bar{f}_{i}$;

2. compute the equilibrium moments;

3. relax the moments according to Eqs. (3.15)-(3.19);

4. reconstruct the distribution functions using Eq. (3.10) and the post-collisional moments.

If the cubic correction is used, $\mathcal{J}_{x}^{(0)}$ and $\mathcal{J}_{y}^{(0)}$ are given by Eqs. (3.11) and (3.12) and $\tau_{x x}$ and $\tau_{y y}$ are given above; otherwise $\mathcal{J}^{(0)}=0$ and $\tau_{x x}=\tau_{y y}=\tau$. Here the relaxation time $\tau_{J}$ is determined according to the value of $\Lambda$ and we set $\tau_{n}=\tau$. Note that $\mathcal{J}_{x}^{(0)}=\mathcal{J}_{y}^{(0)}=0$ at noslip boundaries and the cubic correction does not interact with the boundary conditions considered here.

\section{Constitutive equation embedded in the LBM}

Eqs. (2.7)-(2.9) derived from the discrete Boltzmann equation using the expansion truncated at first order in $\tau$ are the Navier-Stokes equations for a weakly compressible Newtonian fluid with an ideal equation of state for the pressure $p=\rho / 3$ and dynamic viscosity $\mu=\rho \tau / 3$. However, the discrete Boltzmann equation (like the continuous Boltzmann equation) predicts further contributions to the stress field beyond the Navier-Stokes order, at $\mathcal{O}\left(\tau^{2}\right)$ - the so-called Burnett order. If we take moments of Eq. (2.1) with respect 
to the peculiar velocity $\mathbf{c}_{i}=\boldsymbol{\xi}_{i}-\mathbf{u}$, the left hand side of Eq. (2.5c) becomes $[12,55,56]$

$$
\begin{aligned}
\partial_{t} \Pi_{\alpha \beta}+\partial_{\gamma} Q_{\alpha \beta \gamma}= & \partial_{t}\left(\Pi_{\alpha \beta}^{(0)}-T_{\alpha \beta}\right) \\
& +\partial_{\gamma}\left[S_{\alpha \beta \gamma}+u_{\alpha}\left(p \delta_{\beta \gamma}-T_{\beta \gamma}\right)+u_{\beta}\left(p \delta_{\gamma \alpha}-T_{\gamma \alpha}\right)\right. \\
& \left.+u_{\gamma}\left(p \delta_{\alpha \beta}-T_{\alpha \beta}\right)+\rho u_{\alpha} u_{\beta} u_{\gamma}\right],
\end{aligned}
$$

where $T_{\alpha \beta}=p \delta_{\alpha \beta}-\sum_{i} c_{i \alpha} c_{i \beta} f_{i}=\Pi_{\alpha \beta}^{(0)}-\Pi_{\alpha \beta}$ is the deviatoric stress tensor, and $S_{\alpha \beta \gamma}=$ $\sum_{i} f_{i} c_{i \alpha} c_{i \beta} c_{i \gamma}$. The mass and momentum conservation equations (2.7) and (2.8) can be used to evaluate $\partial_{t}\left(\rho u_{\alpha} u_{\beta}\right)$, which arises from the temporal derivative of $\Pi_{\alpha \beta}^{(0)}$ on the right-hand side of (4.1). Following Dellar [12] we assume the collision operator is constructed so that the relaxation time for third order moment is small compared to the stress relaxation time so that it is always close to equilibrium, but we do not neglect the $\mathcal{O}\left(M a^{2}\right)$ and smaller terms. This yields the LBM deviatoric stress

$$
\begin{aligned}
& T_{\alpha \beta}+\tau\left[\partial_{t} T_{\alpha \beta}+u_{\gamma} \partial_{\gamma} T_{\alpha \beta}+T_{\alpha \gamma} \partial_{\gamma} u_{\beta}+T_{\beta \gamma} \partial_{\gamma} u_{\alpha}+T_{\alpha \beta} \partial_{\gamma} u_{\gamma}\right] \\
= & \mu\left(\partial_{\beta} u_{\alpha}+\partial_{\alpha} u_{\beta}\right)-\tau \partial_{\gamma}\left(\rho u_{\alpha} u_{\beta} u_{\gamma}\right) .
\end{aligned}
$$

Eq. (4.2) is the equation for stress that is embedded within the lattice Boltzmann model and it is clearly non-Newtonian (for the incompressible Navier-Stokes equations $T_{\alpha \beta}=$ $\left.\mu\left(\partial_{\beta} u_{\alpha}+\partial_{\alpha} u_{\beta}\right)\right)$. Thus one expects to see LBM computations for the stress to depart from the Navier-Stokes stress when $\tau$ is small (i.e. when $K n \propto M a / R e \gg 0$ ). Eq. (4.2) resembles the Upper Convected Maxwell (UCM) model of viscoelasticity [57] but there is a compressible term and an $\mathcal{O}\left(M a^{3}\right)$ term, and the third and fourth terms in the square brackets have opposite signs in the UCM model. Lattice Boltzmann boundary conditions that do not consider the underlying moment PDE system may be vulnerable to additional numerical errors and instabilities.

\subsection{Wall stress}

We discuss here the wall stresses at a moving boundary with particular reference to the upper horizontal wall of the regularised lid-driven cavity flow discussed in Section 1. That is, where

$$
\left(u_{x}(x, 1), u_{y}(x, 1)\right)=\left(16 x^{2}(1-x)^{2}, 0\right), \quad 0 \leq x \leq 1,
$$

in a box $0 \leq x, y \leq 1$ with no-slip walls. Similar expressions can be derived easily for other boundaries and the conditions are simplified for stationary walls or walls moving with a constant velocity. We assume the flow at the wall is quasi-steady (so we can neglect the temporal derivative of T) and near incompressibility. Then the components of the deviatoric stress (4.2) at the boundaries are

$$
\begin{aligned}
& T_{x x}+\tau\left[u_{x} \partial_{x} T_{x x}+2 T_{x x} \partial_{x} u_{x}+2 T_{x y} \partial_{y} u_{x}\right]=2 \mu \partial_{x} u_{x}-\tau \partial_{x}\left(\rho u_{x}^{3}\right), \\
& T_{x y}+\tau\left[u_{x} \partial_{x} T_{x y}+T_{x y} \partial_{y} u_{y}+T_{x y} \partial_{x} u_{x}+T_{y y} \partial_{y} u_{y}\right]=\mu \partial_{y} u_{x} \\
& T_{y y}+\tau\left[u_{x} \partial_{x} T_{y y}+2 T_{y y} \partial_{y} u_{y}\right]=2 \mu \partial_{y} u_{y} .
\end{aligned}
$$


Since we assume $\nabla \cdot \mathbf{u}=0$ at the wall and we are allowing for the case $u=u(x), \partial_{y} u_{y}=$ $-\partial_{x} u_{x}$ at the wall. Furthermore, to consistent order the convective term in Eq. (4.6) is $\tau u_{x} \partial_{x} T_{y y}=2 \tau \mu u_{x} \partial_{y} u_{y}=-2 \tau \mu u_{x} \partial_{x} u_{x}$, which can be computed exactly since $u=u(x)$ is given. Thus, the normal component of the stress is

$$
T_{y y}=\frac{2 \mu\left(\tau u_{x} \partial_{x x} u_{x}-\partial_{x} u_{x}\right)}{1-2 \partial_{x} u_{x}}
$$

Note that in the case of a boundary moving with a constant velocity, $T_{y y}=0$. By similar arguments, the off-diagonal component becomes

$$
T_{x y}=\tau \partial_{y} u_{x}\left(\frac{\rho}{3}-T_{y y}\right)=\tau \Pi_{y y} \partial_{y} u_{x}
$$

where we have used the fact that $\partial_{x y} u_{x}=-\partial_{y y} u_{y}$, by the continuity condition, and made the approximation that this is zero since the velocity near the boundary is predominately in the $x$ direction. Note that if $u_{x}$ is constant then $T_{x y}=\mu \partial_{y} u_{x}$, which is the Newtonian shear stress at the wall. Finally, we find an expression for the tangential component of deviatoric stress at the moving boundary. We will drop the $\mathcal{O}\left(M a^{3}\right)$ term for the time being and note that to consistent order the convective term in Eq. (4.4) is $\tau u_{x} \partial_{x} T_{x x}=$ $\tau u_{x}=2 \tau \mu u_{x} \partial_{x x} u_{x}$. Then rearranging (4.4) gives

$$
T_{x x}=\frac{2 \mu\left(\partial_{x} u_{x}-\tau u_{x} \partial_{x x} u_{x}\right)-2 \frac{T_{x y}^{2}}{\Pi_{y y}}}{1+2 \tau \partial_{x} u_{x}} .
$$

All terms in Eq. (4.9) can be computed exactly and locally at grid points. Note that the tangential stress at the boundary reduces to $T_{x x}=-6 T_{x y}^{2} \rho=-\mu \tau\left(\partial_{y} u_{x}\right)^{2}$ when $\partial_{x} u_{x}=0$.

When the cubic correction discussed in Section 3.2 is implemented and $\tau$ is replaced by $\tau_{x x}=\tau\left(1-9 / 2 u_{x}^{2}\right)^{-1}$ the constitutive equation (4.4) becomes

$$
\begin{aligned}
& T_{x x}+\frac{\tau}{1-9 / 2 u_{x}^{2}}\left(u_{x} \partial_{x} T_{x x}+2 T_{x x} \partial_{x} u_{x}+2 T_{x y} \partial_{y} u_{x}\right) \\
= & \frac{\tau}{1-9 / 2 u_{x}^{2}}\left(\frac{2}{3} \rho \partial_{x} u_{x}-\partial_{x}\left(\rho u_{x}^{3}\right)\right) \\
= & \frac{\tau}{1-9 / 2 u_{x}^{2}}\left(\frac{2}{3} \rho \partial_{x} u_{x}-3 \rho u_{x}^{2} \partial_{x} u_{x}-u_{x}^{3} \partial_{x} \rho\right) \\
= & \tau\left(\frac{2}{3} \rho \partial_{x} u_{x}-\frac{u_{x}^{3}}{1-9 / 2 u_{x}^{2}} \partial_{x} \rho\right) .
\end{aligned}
$$

That is, the right hand side of Eq. (4.10) is the same as the right hand side of Eq. (3.14) and the non-Galilean invariance error of Eq. (4.9) is reduced by two orders of magnitude in Mach number. 


\section{Moment-based boundary conditions}

We consider solid boundaries that are located precisely on lattice nodes. After each streaming step the distribution functions at a boundary node with particle velocities pointing into the fluid are not known. To fix ideas, imagine a horizontal boundary located along the top row of grid points. Here, $\bar{f}_{4}, \bar{f}_{7}$ and $\bar{f}_{8}$ (see Fig. 2 ) are unknown after each streaming step and must be supplied by the boundary conditions. All other $\bar{f}_{i}$ at the boundary are known from the algorithm. To find the three unknown $\bar{f}_{i}$ we need three linear independent equations.

While the Boltzmann equation in continuous kinetic theory has an infinite hierarchy of velocity moments, the lattice Boltzmann equation has an invertible relation between the $\bar{f}_{i}$ and a finite set of independent moments. Thus one may consider specifying boundary conditions for a sub-set of moments that suffice to determine the unknown $\bar{f}_{i}$. To decide which moments one should impose boundary conditions upon, Bennett [32] listed the combinations of the unknown distribution functions that contribute to each moment at a boundary. To illustrate the principle, we look at how $\bar{f}_{4}, \bar{f}_{7}$ and $\bar{f}_{8}$ are arranged in each moment at the upper boundary. The zeroth-order moment (density), for example, may be written as $\rho=\left(\bar{f}_{4}+\bar{f}_{7}+\bar{f}_{8}\right)+\bar{f}_{0}+\bar{f}_{1}+\bar{f}_{2}+\bar{f}_{3}+\bar{f}_{5}+\bar{f}_{6}$, where the bracketed $\bar{f}_{i}$ are unknown. Table 1 shows which combination of the three unknowns appear in each of the nine moments, including the fourth-order moment defined by $\bar{R}_{x x y y}=\sum_{i} \bar{f}_{i} \xi_{x}^{2} \xi_{y}^{2}$. Moments in different rows of Table 1 are linearly independent.

Table 1: Moment groups at the upper boundary.

\begin{tabular}{||c|c||}
\hline Moments & Combination of unknowns \\
\hline \hline$\rho, \rho u_{y}, \bar{\Pi}_{y y}$ & $\bar{f}_{4}+\bar{f}_{7}+\bar{f}_{8}$ \\
\hline$\rho u_{x}, \bar{\Pi}_{x y}, \bar{Q}_{x y y}$ & $\bar{f}_{8}-\bar{f}_{7}$ \\
\hline $\bar{\Pi}_{x x}, \bar{Q}_{x x y}, \bar{R}_{x x y y}$ & $\bar{f}_{7}+\bar{f}_{8}$ \\
\hline
\end{tabular}

To determine the three unknown variables we must choose one moment from each row of Table 1 , impose constraints on these moments, and then solve for $\bar{f}_{4}, \bar{f}_{7}$, and $\bar{f}_{8}$. It is natural to choose the hydrodynamic moments rather than higher-order moments that do not appear in the governing equations. To ensure no slip and no flux at the boundary, we simply set $u_{x}=u_{x}^{w}$ and $u_{y}=0$, where $u_{x}^{w}$ is the velocity of the upper boundary. The only other linearly independent hydrodynamic moment in Table 1 is $\bar{\Pi}_{x x}$. Therefore we impose a physically suitable boundary condition on $\Pi_{x x}$, and convert it into a condition on $\bar{\Pi}_{x x}$ using Eq. (3.6).

The boundary condition for the tangential component of $\Pi$ requires some attention. The original moment-based implementation, which is used in all related works, sets $\Pi_{x x}=\Pi_{x x}^{(0)}$ (equivalent to $T_{x x}=0$ ), which is justified by noting that $\Pi_{x x} \sim \Pi_{x x}^{(0)}+\tau \Pi_{x x}^{(1)}$ and $\Pi_{x x}^{(1)} \propto \partial_{x} u_{x}=0$ along a boundary (cf. Eq. (3.13)). However, the velocity of the top wall 
is not constant in the regularized cavity (see Eq. (1.1)). Moreover, the lattice Boltzmann deviatoric stress is actually governed by Eq. (4.2) which produces an $\mathcal{O}\left(\tau^{2}\right)$ Burnett contribution. Thus the original Navier-Stokes condition on $\Pi_{x x}$ is not consistent with the underlying PDE moment system that the LBM is computing. Section 4.1 showed how one can find consistent conditions for the deviatoric stress $\mathbf{T}$, for example Eq. (4.9) for a wall moving with velocity $\mathbf{u}=\left(u_{x}(x), 0\right)$. These can be formulated as conditions on $\boldsymbol{\Pi}=\boldsymbol{\Pi}^{(0)}-\mathbf{T}$, and thus on $\overline{\boldsymbol{\Pi}}$ and the unknown $\bar{f}_{i}$.

The boundary condition for $\Pi_{x x}$ at the upper boundary of the flow considered here is $\Pi_{x x}=\Pi_{x x}^{(0)}-T_{x x}=\rho / 3+\rho u_{x}^{2}-T_{x x}$, where the deviatoric stress is given by Eq. (4.9) and $u_{x}=16 x^{2}(1-x)^{2}$ is the imposed tangential velocity of the boundary. In view of the transformation (3.3) we formulate the stress boundary condition as

$$
\begin{aligned}
\bar{\Pi}_{x x} & =\frac{2 \tau+\Delta t}{2 \tau} \Pi_{x x}-\frac{\Delta t}{2 \tau} \Pi_{x x}^{(0)} \\
& =\frac{\rho}{3}+\rho u_{x}^{2}-\frac{2 \tau+\Delta t}{2 \tau} T_{x x} .
\end{aligned}
$$

The three moment equations $\rho u_{x}, \rho u_{y}$, and $\bar{\Pi}_{x x}$ with their respective boundary conditions are easily solved to find the unknown distribution functions,

$$
\begin{aligned}
& \bar{f}_{4}=\bar{f}_{1}+\bar{f}_{2}+\bar{f}_{3}+2 \bar{f}_{5}+2 \bar{f}_{6}-\bar{\Pi}_{x x}, \\
& \bar{f}_{7}=-\bar{f}_{3}-\bar{f}_{6}-\frac{1}{2}\left(u_{x}-\bar{\Pi}_{x x}\right), \\
& \bar{f}_{8}=-\bar{f}_{1}-\bar{f}_{5}+\frac{1}{2}\left(u_{x}+\bar{\Pi}_{x x}\right) .
\end{aligned}
$$

The expression for $\bar{\Pi}_{x x}$ above contains the density at the boundary. This can be found in terms of the velocity and the known distribution functions using

$$
\rho=\sum_{i} \bar{f}_{i}=-\rho u_{y}+\bar{f}_{0}+\bar{f}_{1}+\bar{f}_{3}+2\left(\bar{f}_{2}+\bar{f}_{5}+\bar{f}_{6}\right),
$$

and the boundary condition $u_{y}=0$. The boundary condition (4.9) for $T_{x x}$, and hence $\bar{\Pi}_{x x}$, involves the diagonal component of the deviatoric stress $T_{x y}$ at the boundary. This is computed locally from known (outgoing) $\bar{f}_{i}$ and the imposed condition on the momentum tangential to the wall. For example, at the top boundary

$$
\begin{aligned}
T_{x y} & =\Pi_{x y}^{(0)}-\Pi_{x y}=-\Pi_{x y}=-\frac{2 \tau+\Delta t}{2 \tau} \bar{\Pi}_{x y} \\
& =\frac{2 \tau+\Delta t}{2 \tau}\left(\rho u_{x}-\bar{f}_{1}+\bar{f}_{3}-2 \bar{f}_{5}+2 \bar{f}_{6}\right) .
\end{aligned}
$$

Similar expressions determine the unknown distribution functions at the other boundaries (note that $u_{T}=\partial_{T} u_{T}=0$ at the three other boundaries, where the subscript $T$ 
denotes the axis-aligned planar tangential component). Condition (4.9) also involves $\Pi_{y y}$, which again can be computed locally. For example, at the top boundary, $\Pi_{y y}=$ $-\rho u_{y}+2\left(f_{2}+f_{5}+f_{6}\right)=2\left(f_{2}+f_{5}+f_{6}\right)$. Converting to "barred" variables for implementation, this becomes

$$
\Pi_{y y}=\frac{6 \tau \bar{\Pi}_{y y}+\rho}{2 \tau+1}
$$

where $\bar{\Pi}_{y y}=2\left(\bar{f}_{2}+\bar{f}_{5}+\bar{f}_{6}\right)$ and $\rho$ at the wall is given by Eq. (5.4).

There are five unknown $\bar{f}_{i}$ at each corner node, so five moment constraints are required. For consistency, we choose the same moments that are used for the adjoining boundaries, i.e. $\rho u_{x}, \rho u_{y}, \bar{\Pi}_{x x}$ and $\bar{\Pi}_{y y}$. For the final constraint we use the off-diagonal component of the deviatoric stress and set $T_{x y}=0$ (see Eq. (4.8)). This is equivalent to imposing $\bar{\Pi}_{x y}=0$ at the corners.

\section{Numerical simulations}

To assess the method with complicated boundary conditions we apply the moment based formulation with the Burnett correction to the stress discussed in Section 5 to the regularized lid-driven cavity problem. We first assess the accuracy of the method by comparing our results with benchmark data obtained using a spectral method for Reynolds numbers in the range $100 \leq R e \leq 5000$, and then study the LBM stress field in this flow at lower $R e$ (and hence higher $K n$ ). The regularised cavity flow is inside the $0 \leq x \leq 1,0 \leq y \leq 1$ box with no-slip boundaries. The upper boundary moves with a steady but spatially-dependent positive horizontal velocity and the three other boundaries remain stationary. The velocity of the upper boundary is given by Eq. (1.1). The velocity of the upper boundary, $u_{x}$, smoothly decays to zero at the corners, which removes the corner stress singularities that occur in the more common benchmark problem with a constant velocity. The maximum velocity at the upper boundary, $U=u_{x}(1 / 2,1)=1$, is used to define the Reynolds number and the particle velocity $c=\Delta x / \Delta t=10$ so that the Mach number based on the maximum velocity of the lid is $M a=\sqrt{3} / 10$.

Table 2 shows the maximum value of the streamfunction on successive meshes computed using the BGK collision operator with and without the cubic correction. The streamfunction $\psi$ is computed from the lattice Boltzmann velocity field by first approximating the vorticity as

$$
\omega=\frac{v(x+\Delta x, y)-v(x-\Delta x, y)}{2 \Delta x}-\frac{u(x, y+\Delta x)-u(x, y-\Delta x)}{2 \Delta x},
$$

and using a multigrid Poisson solver to obtain $\psi$ from $\nabla^{2} \psi=-\omega$ with $\psi=0$ on the boundary [58]. The table shows the smallest grids that were able to maintain stability at different Reynolds numbers for grids of size $N^{2}$, where $N=2^{n}+1, n \in \mathbb{N}$ (note that $\Delta x=1 /(N-1)$ ), and the spectral results of Shen [44] are shown for comparison. The stress boundary conditions and the removal of the corner singularities enable us to simulate the flow up to a 
Table 2: Resolution dependence of the primary streamfunction computed with the moment method using the BGK collision operator. Results are shown with and without the cubic correction.

\begin{tabular}{||c||c|c||}
\hline & Without cubic correction & With cubic correction \\
\hline$R e=100$ & $\psi_{P}$ & $\psi_{P}$ \\
$N=33$ & 0.08342 & 0.08330 \\
$N=65$ & 0.08364 & 0.08355 \\
$N=129$ & 0.08366 & 0.08358 \\
$N=257$ & 0.08368 & 0.08359 \\
$N=513$ & 0.08368 & 0.08360 \\
Shen [44] & 0.08368 & 0.08368 \\
\hline$R e=400$ & $\psi_{P}$ & $\psi_{P}$ \\
$N=129$ & 0.08529 & 0.08575 \\
$N=257$ & 0.08581 & 0.08581 \\
$N=513$ & 0.08589 & 0.08582 \\
Shen [44] & 0.08584 & 0.08584 \\
\hline$R e=1000$ & $\psi_{P}$ & $\psi_{P}$ \\
$N=257$ & 0.08710 & 0.08703 \\
$N=513$ & 0.08720 & 0.08713 \\
Shen [44] & 0.08719 & 0.08719 \\
\hline
\end{tabular}

Reynolds number of 1000 on reasonably sized grids. This could not be achieved with the Navier-Stokes condition with the moment-based boundary conditions for the classical lid driven cavity flow [39]. Thus the removal of the stress singularity with the consistent condition does improve the stability of the algorithm, but we still need more grid points than the bounce-back method to reach high Reynolds numbers (in [41], only $129^{2}$ grid points were needed to obtain sensible predictions when $R e=1000$ using a BGK collision operator with bounce-back, and this was using the standard lid-driven cavity flow). Therefore we conclude that although the removal to singularities with the consistent stress conditions offers improvements in terms of stability, there are other factors that influence the stability of the moment-based approach. This is a topic for future research. The accuracy of the results in comparison with spectral method computations is excellent.

Table 3 shows the maximum value of the streamfunction $\psi$ and its location computed with TRT LBE using the two special choices $\Lambda=1 / 4$ and $\Lambda=1 / 6$. The effect of the grid resolution $N$ is also shown (note that $\Delta x=1 /(N-1)$ ). Cells in the tables with "-" indicate unstable simulations. Simulation results are shown with and without the cubic correction described in Section 3.2. Clearly the LBM with moment-based boundary conditions and a TRT operator with $\Lambda=1 / 4$ is very stable, allowing us to reach high Reynolds numbers on coarser meshes than the BGK model. When $\Lambda=1 / 6$ the stability properties appear to be similar to the BGK case. The computations show good qualitative agreement with the known flow characteristics, including the primary vortex towards the centre of the cavity, 
Table 3: Resolution dependence of the primary streamfunction computed with the moment method using different values of the magic parameter. Results are shown with and without the cubic correction.

\begin{tabular}{|c|c|c|c|c|c|c|c|c|c|c|c|c|}
\hline & \multicolumn{6}{|c|}{ Without cubic correction } & \multicolumn{6}{|c|}{ With cubic correction } \\
\hline & \multicolumn{3}{|c|}{$\Lambda=1 / 4$} & \multicolumn{3}{|c|}{$\Lambda=1 / 6$} & \multicolumn{3}{|c|}{$\Lambda=1 / 4$} & \multicolumn{3}{|c|}{$\Lambda=1 / 6$} \\
\hline$R e=100$ & $\psi_{P}$ & $x$ & $y$ & $\psi_{P}$ & $x$ & $y$ & $\psi_{P}$ & $x$ & $y$ & $\psi_{P}$ & $x$ & $y$ \\
\hline$N=17$ & 0.08436 & 0.6250 & 0.7500 & - & - & - & 0.08595 & 0.6250 & 0.7500 & - & - & - \\
\hline$N=33$ & 0.08353 & 0.5938 & 0.7500 & 0.08253 & 0.5938 & 0.7500 & 0.08348 & 0.5938 & 0.7500 & 0.08245 & 0.5938 & 0.7500 \\
\hline$N=65$ & 08365 & 0.6094 & 0.7500 & 0.08361 & 0.6094 & 0.7500 & 0.08356 & 0.6094 & 0.7500 & 0.08353 & 0.6904 & 0.7500 \\
\hline$N=129$ & 0.08367 & 0.6094 & 0.7578 & 0.08366 & 0.6094 & 0.7578 & 0.08358 & 0.6094 & 0.7578 & 0.08358 & 0.6094 & 0.7578 \\
\hline$N=257$ & 0.08368 & 0.6055 & 0.7539 & 0.08368 & 0.6094 & 0.7539 & 0.08359 & 0.6055 & 0.7539 & 0.08359 & 0.6055 & 0.7539 \\
\hline$N=513$ & 08368 & 0.6074 & 0.7539 & 0.08368 & 0.6074 & 0.7539 & 0.08359 & 0.6074 & 0.7539 & 0.08359 & 0.6074 & 0.7539 \\
\hline$R e=400$ & $\psi_{P}$ & $x$ & $y$ & $\psi_{P}$ & $x$ & $y$ & $\psi_{P}$ & $x$ & $y$ & $\psi_{P}$ & $x$ & $y$ \\
\hline$N=17$ & 0.09613 & 0.5625 & 0.6250 & - & - & - & - & - & - & - & - & - \\
\hline$N=33$ & 0.08583 & 0.5938 & 0.6250 & - & - & - & - & - & - & - & - & - \\
\hline$N=65$ & 0.08565 & 0.5781 & 0.6094 & - & - & - & 0.08567 & 0.5781 & 0.6094 & - & - & - \\
\hline$N=129$ & 0.08584 & 0.5781 & 0.6172 & 0.08578 & 0.5781 & 0.6172 & 0.08576 & 0.5781 & 0.6172 & 0.08571 & 0.5781 & 0.6172 \\
\hline$N=257$ & 0.08589 & 0.5781 & 0.6172 & 0.08589 & 0.5781 & 0.6172 & 0.08581 & 0.5781 & 0.6172 & 0.08581 & 0.5781 & 0.6172 \\
\hline$N=513$ & 0.08590 & 0.5781 & 0.6172 & 0.085917 & 0.5781 & 0.6172 & 0.08582 & 0.5781 & 0.6172 & 0.08582 & 0.5781 & 0.6172 \\
\hline$R e=1000$ & $\psi_{P}$ & $x$ & $y$ & $\psi_{P}$ & $x$ & $y$ & $\psi_{P}$ & $x$ & $y$ & $\psi_{P}$ & $x$ & $y$ \\
\hline$N=17$ & 0.1204 & 0.5625 & 0.5625 & - & - & - & - & - & - & - & - & - \\
\hline$N=33$ & 0.08898 & 0.5313 & 0.5625 & - & - & - & - & - & - & - & - & - \\
\hline$N=65$ & 0.08642 & 0.5469 & 0.5781 & - & - & - & - & - & - & - & - & - \\
\hline$N=129$ & 0.08692 & 0.5391 & 0.5703 & - & - & - & 0.08690 & 0.5391 & 0.5703 & - & - & - \\
\hline$N=257$ & 0.08714 & 0.5430 & 0.5742 & 0.08712 & 0.5430 & 0.5742 & 0.08707 & 0.5430 & 0.5742 & 0.08705 & 0.5430 & 0.5742 \\
\hline$N=513$ & 0.08720 & 0.5430 & 0.5723 & 0.08720 & 0.5430 & 0.5723 & 0.08713 & 0.5430 & 0.5723 & 0.08713 & 0.5430 & 0.5723 \\
\hline$R e=5000$ & $\psi_{P}$ & $x$ & $y$ & $\psi_{P}$ & $x$ & $y$ & $\psi_{P}$ & $x$ & $y$ & $\psi_{P}$ & $x$ & $y$ \\
\hline$N=65$ & 0.07509 & 0.5156 & 0.5313 & - & - & - & - & - & - & - & - & - \\
\hline$N=129$ & 0.08514 & 0.5156 & 0.5391 & - & - & - & - & - & - & - & - & - \\
\hline$N=257$ & 0.08732 & 0.5195 & 0.5391 & - & - & - & - & - & - & - & - & - \\
\hline$N=513$ & 0.08794 & 0.5195 & 0.5391 & 0.08795 & 0.5195 & 0.5391 & 0.08788 & 0.5195 & 0.5391 & 0.08789 & 0.5195 & 0.5391 \\
\hline
\end{tabular}

and two smaller vortices near the lower corners. A further vortex is found in the upper righthand corner when $R e=5000$. Fig. 3 shows the streamfunction and the pressure when $R e=5000$ and $\Lambda=1 / 4$.

To assess the convergence and order of accuracy of the moment method we compute the global relative error norms

$$
\|\Xi\|_{2}=\frac{\sum_{\mathbf{x}}\left\|\Xi(\mathbf{x})-\Xi_{r e f}(\mathbf{x})\right\|_{2}}{\sum_{\mathbf{x}}\left\|\Xi_{r e f}(\mathbf{x})\right\|_{2}}, \quad \Xi \in\{\mathbf{u}, p\},
$$

where $\Xi_{r e f}$ is the reference solution and $\|\cdot\|_{2}$ denotes the Euclidean $L_{2}$ norm. Fig. 4 plots the relative error norm for velocity and pressure at a Reynolds number of $R e=1000$ using $\Lambda=1 / 4$. Both fields show second-order convergence, confirming the accuracy of the moment based boundary conditions. We have studied the convergence for different Reynolds number and observed the same order of convergence in all cases. For completeness, results for the standard lid driven cavity test with a constant upper boundary velocity are given in Appendix A. 

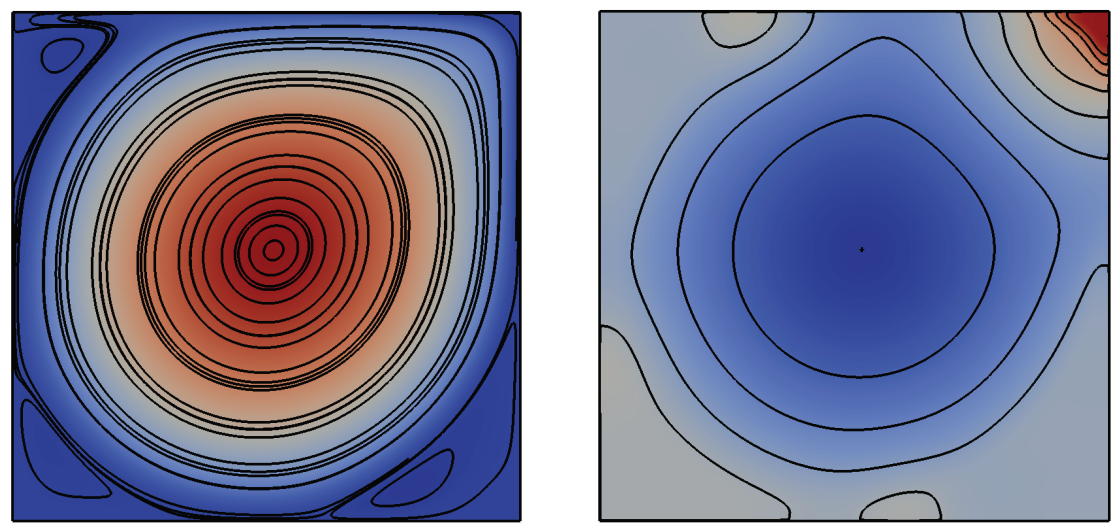

Figure 3: The streamfunction (left) and pressure at $R e=5000$ computed with the moment method and $\Lambda=1 / 4$.

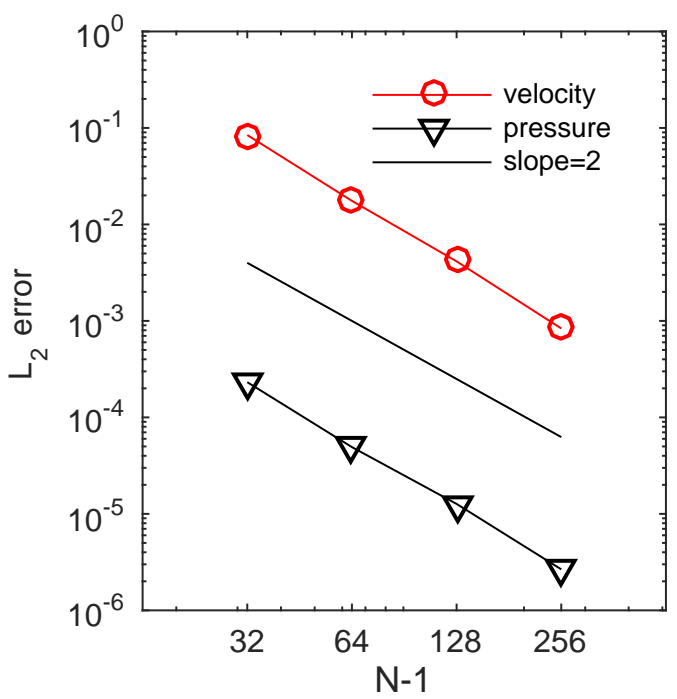

Figure 4: Plot showing second-order convergence of velocity and pressure for $\operatorname{Re}=1000$ and $\Lambda=1 / 4$.

Table 4 compares the results computed by the moment method with $\Lambda=1 / 4$ with the benchmark data of Shen [44]. Shown are comparisons of the value of the streamfunction at the centre of the primary, lower left, lower right, and upper left vortices, and its location. The lattice Boltzmann computations are in excellent agreement with the results from the spectral method of Shen [44], confirming the validity of the proposed model.

\subsection{Computations of the stress at low Reynolds numbers}

In this subsection we inspect the LBM deviatoric stress at smaller Re in the same flow to highlight its non-Newtonian behaviour. The grid size in all cases is $129^{2}$ and results are 
Table 4: Comparison of the streamfunction computed with the moment method and TRT collisions with the spectral element results of Shen [44]. There is no vortex in the upper left hand corner for $\operatorname{Re} \in\{100,400,1000\}$.

\begin{tabular}{|c|c|c|c|c|c|c|c|c|c|c|c|c|}
\hline & \multicolumn{3}{|c|}{ Primary } & \multicolumn{3}{|c|}{ Left } & \multicolumn{3}{|c|}{ right } & \multicolumn{3}{|c|}{ upper } \\
\hline$R e=100$ & $\psi_{P}$ & $x$ & $y$ & $\psi_{L}$ & $x$ & $y$ & $\psi_{R}$ & $x$ & $y$ & $\psi_{U}$ & $x$ & $y$ \\
\hline$\Lambda=1 / 4$ & 0.08368 & 0.6250 & 0.7500 & $-1.3569 \times 10^{-6}$ & 0.033 & 0.035 & $-4.8811 \times 10^{-6}$ & 0.953 & 0.049 & & & \\
\hline Cubic, $\Lambda=1 / 4$ & 0.08359 & 0.6250 & 0.7500 & $-1.3561 \times 10^{-6}$ & 0.033 & 0.035 & $-4.8745 \times 10^{-6}$ & 0.953 & 0.049 & & & \\
\hline Shen [44] & 0.08368 & 0.609 & 0.750 & $-1.3987 \times 10^{-6}$ & 0.031 & 0.031 & $-4.6676 \times 10^{-6}$ & 0.953 & 0.047 & & & \\
\hline$R e=400$ & & & & & & & & & & & & \\
\hline$\Lambda=1 / 4$ & 0.08590 & 0.5781 & 0.6172 & $-5.6405 \times 10^{-6}$ & 0.045 & 0.043 & $-2.5655 \times 10^{-4}$ & 0.900 & 0.113 & & & \\
\hline Cubic, $\Lambda=1 / 4$ & 0.08582 & 0.5781 & 0.6172 & $-5.6302 \times 10^{-6}$ & 0.045 & 0.043 & $-2.5613 \times 10^{-4}$ & 0.900 & 0.113 & & & \\
\hline Shen [44] & 0.08584 & 0.578 & 0.625 & $-6.63146 \times 10^{-6}$ & 0.031 & 0.047 & $-1.9774 \times 10^{-4}$ & 0.922 & 0.094 & & & \\
\hline$R e=1000$ & & & & & & & & & & & & \\
\hline$\Lambda=1 / 4$ & 0.08720 & 0.5430 & 0.5723 & $-8.3586 \times 10^{-5}$ & 0.078 & 0.068 & $-9.8733 \times 10^{-4}$ & 0.871 & 0.115 & & & \\
\hline Cubic, $\Lambda=1 / 4$ & 0.08713 & 0.5430 & 0.5723 & $-8.3513 \times 10^{-5}$ & 0.078 & 0.068 & $-9.8657 \times 10^{-4}$ & 0.871 & 0.115 & & & \\
\hline Shen [44] & 0.08719 & 0.547 & 0.578 & $-8.2841 \times 10^{-5}$ & 0.078 & 0.063 & $-5.67626 \times 10^{-4}$ & 0.922 & 0.094 & & & \\
\hline$R e=5000$ & & & & & & & & & & & & \\
\hline$\Lambda=1 / 4$ & 0.08794 & 0.5195 & 0.5391 & $-8.3731 \times 10^{-4}$ & 0.080 & 0.123 & $-2.1133 \times 10^{-3}$ & 0.814 & 0.082 & $-7.7211 \times 10^{-4}$ & 0.085 & 0.912 \\
\hline Cubic, $\Lambda=1 / 4$ & 0.08788 & 0.5195 & 0.5391 & $-8.3742 \times 10^{-4}$ & 0.080 & 0.123 & $-2.1129 \times 10^{-3}$ & 0.814 & 0.082 & $-7.7192 \times 10^{-4}$ & 0.085 & 0.912 \\
\hline Shen [44] & 0.08803 & 0.516 & 0.531 & $-7.5268 \times 10^{-4}$ & 0.094 & 0.094 & $-7.7475 \times 10^{-4}$ & 0.922 & 0.094 & $-6.7780 \times 10^{-4}$ & 0.078 & 0.092 \\
\hline
\end{tabular}

independent of further mesh resolution. The particle velocity $c=\Delta x / \Delta t=10$ so that the Mach number based on the maximum velocity of the lid is $M a=\sqrt{3} / 10$ in all computations in this section. The tangential deviatoric stresses are non-dimensional and scaled by $c^{2}$, and the graphs that follow are plotted every 6 data points. The TRT collision operator with "magic parameter" $\Lambda=1 / 4$ was used in all simulations. This is not so much because of stability but because when $R e$ is small, the relaxation time $\tau$ is large (unless we have fine spatial and temporal discretisations), but the analysis discussed in Section 4 requires the relaxation time of the third order moment $Q_{\alpha \beta \gamma}=\sum_{i} f_{i} c_{i \alpha} c_{i \beta} c_{i \gamma}$ to be small so that $Q_{\alpha \beta \gamma} \sim Q_{\alpha \beta \gamma}^{(0)}$. This is achievable with TRT $\Lambda=\tau \tau_{J}=1 / 4$. Different collision models with $\tau_{J}>1$ and $\tau<1$ can lead to very different predications for the stress because the moment $Q_{\alpha \beta \gamma}$ decays very slowly its equilibrium and becomes a "quasi conserved" quantity.

Fig. 5 plots the tangential component of the deviatoric stress, $T_{x x}(x, 1 / 2)$, and the viscous stress, $\sigma_{x x}(x, 1 / 2)=\left.\mu \partial_{x} u_{x}\right|_{y=1 / 2}$, on the horizontal through the centre of the cavity using the TRT LBM when $R e=10$ (left plot). The Knudsen number is $K n \sim M a / R e=$ $\sqrt{3} / 100$. The velocity gradients are zero at the wall and near centre of the flow and both stresses are largest in magnitude near a quarter length away from the boundaries. The tangential component of the deviatoric stress, $T_{x x}(1 / 2, y)$, and the viscous stress, $\sigma_{x x}(1 / 2, y)$, on the vertical through the centre of the cavity are shown in the plot on the right in Fig. 5 and there are discernible differences near the moving wall, where we have imposed the Burnett stress condition. The differences between the LBM stress and viscous stress in both plots is difficult to discern because they are of size $\tau^{2}$.

When the Reynolds number is lowered to $R e=0.5(K n \sim M a / R e=\sqrt{3} / 5)$ we can see from the left hand plot in Fig. 6 that $T_{x x}(x, 1 / 2)$ and $\sigma_{x x}(x, 1 / 2)$ are still very similar but with small differences due to the Burnett contributions to $T_{x x}$ becoming visible. The difference between the LBM and Newtonian stress is far more prominent along the vertical 

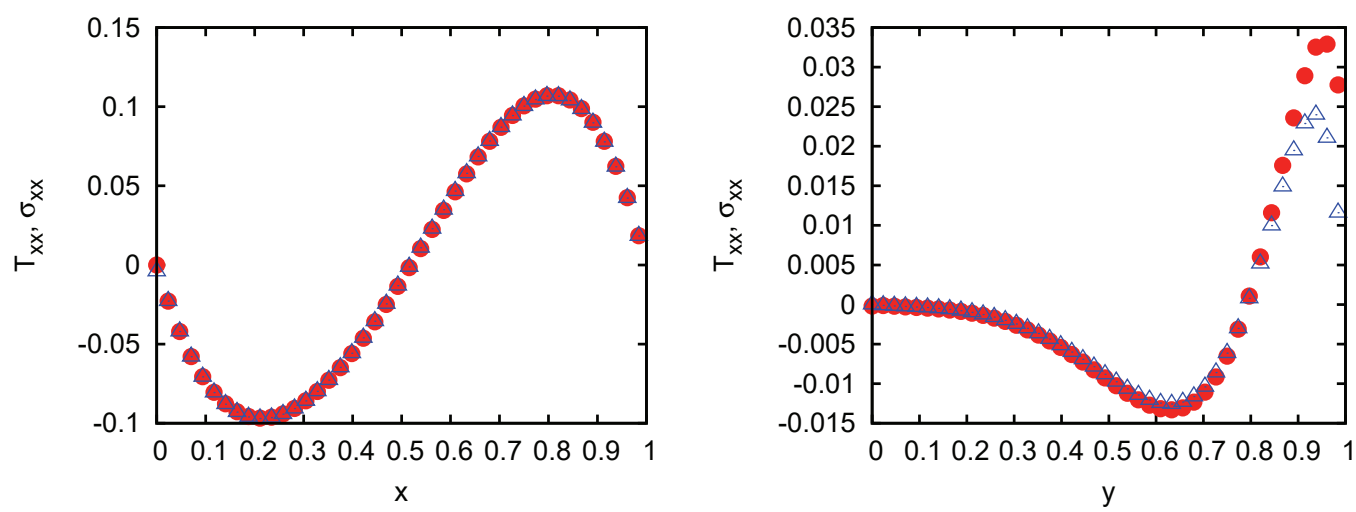

Figure 5: Plot showing (left) the deviatoric stress $T_{x x}(x, 1 / 2)$ (circles) and the Newtonian viscous stress $\sigma_{x x}(x, 1 / 2)$ along the horizontal centreline, and (right) $T_{x x}(1 / 2, y)$ (circles) and $\sigma_{x x}(1 / 2, y)$ along the vertical centreline computed from the LBM when $R e=10$.
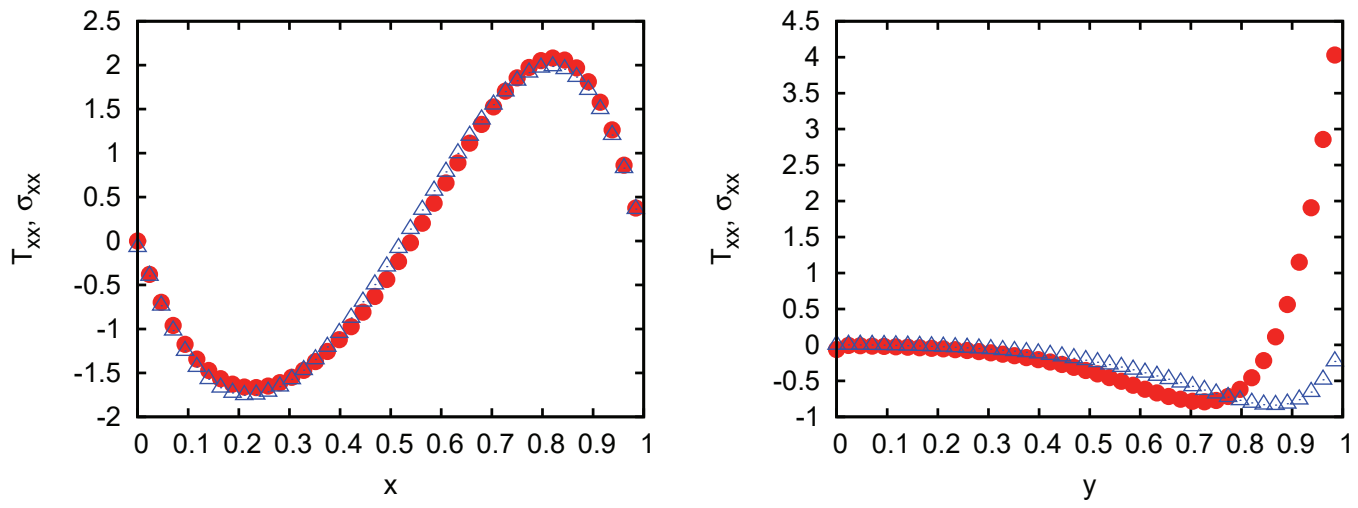

Figure 6: Plot showing (left) the deviatoric stress $T_{x x}(x, 1 / 2)$ (circles) and the Newtonian viscous stress $\sigma_{x x}(x, 1 / 2)$ along the horizontal centreline, and (right) $T_{x x}(1 / 2, y)$ (circles) and $\sigma_{x x}(1 / 2, y)$ along the vertical centreline computed from the LBM when $R e=0.5$.

centreline, which includes the moving boundary, as seen on the plot on the right in Fig. 6. The tangential components of the stresses are very similar in the bottom half of the domain but nearer the moving boundary they are strikingly different: the Newtonian stress at $(1 / 2,1)$ is $\sigma_{x x}=\left.2 \mu \partial_{x} u_{x}\right|_{(1 / 2,1)}=0$ but the LBM derivatoric stress contains non vanishing terms. Dropping the Reynolds number still further to $\operatorname{Re}=0.1(K n \sim M a / \operatorname{Re}=\sqrt{3})$ highlights the fact that the LBM computes a non-Newtonian stress, as shown in Fig. 7, where $T_{x x}(x, 1 / 2)$ has noticeably different turning points to $\sigma_{x x}(x, 1 / 2)$. Along the vertical centreline the Newtonian tangential stress, $\sigma_{x x}(1 / 2, y)$ has a local minima close the movings boundary, at $y \sim 0.9$, but this local minimum is moved towards the centre of the domain in the LBM deviatoric stress. Thus it is clear that although the LBM does not include kinetic effects in the velocity it does have appreciable Burnett contributions in the 

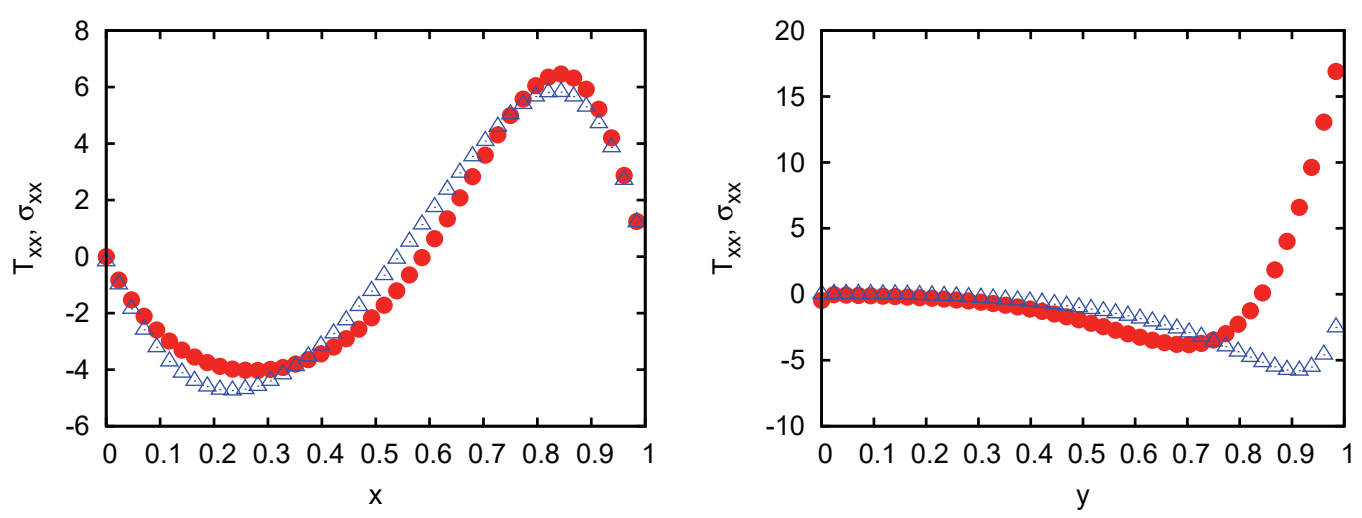

Figure 7: Plot showing (left) the deviatoric stress $T_{x x}(x, 1 / 2)$ (circles) and the Newtonian viscous stress $\sigma_{x x}(x, 1 / 2)$ along the horizontal centreline, and (right) $T_{x x}(1 / 2, y)$ (circles) and $\sigma_{x x}(1 / 2, y)$ along the vertical centreline computed from the LBM when $R e=0.1$.
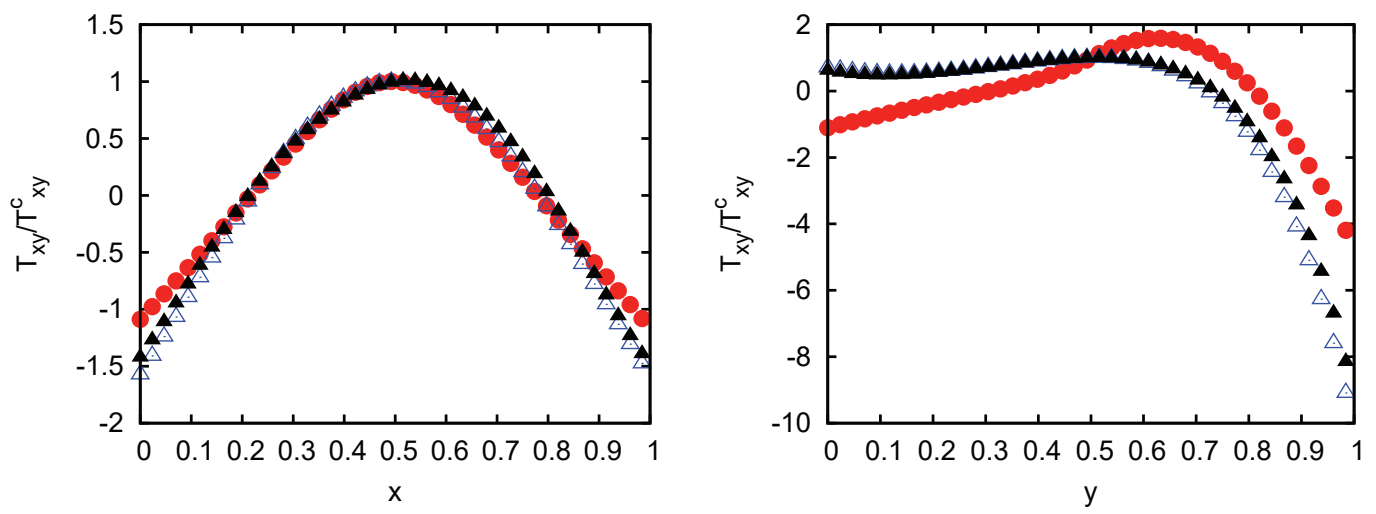

Figure 8: Plot showing the normalised shear stress along the horizontal centreline, $T_{x y}(x, 1 / 2)$ (left) and vertical centreline, $T_{x y}(1 / 2, y)$. Filled triangles are for $R e=10$, empty triangles for $R e=0.5$, and circles for $R e=0.1$.

stress at $M a / R e \gg 0$ that are usually neglected. They diminish in the diffusive scaling, where $M a \rightarrow 0$ under grid refinement such that $\Delta t \propto \Delta x^{2}$, but not in the acoustic scaling with a fixed Mach number. Furthermore, for rarefied flow with non-vanishing Mach and Knudsen numbers, the contributions to $\mathbf{T}$ at Burnett order found from the LBM are present and non negligible. They are embedded within the moments of the LBM and if boundary conditions are to be imposed on any component of the stress then they should be consistent with the PDE the algorithm is solving. We note again that nothing has been added to the LBM to compute the deviatoric stress, $T_{x x}$, other than ensuring consistent boundary conditions are imposed.

Finally, the normalised LBM shear stresses, $T_{x y} / T_{x y}^{c}$ where $T_{x y}^{c}=T_{x y}(1 / 2,1 / 2)$, at $R e=10,0.5$, and 0.1 are shown in Fig. 8. The plot on the left shows the stresses along the horizontal centreline, and the plot on the right is along the vertical centreline. Along the 
horizontal centreline the shear stress near boundaries vary considerably with Reynolds number and the profile of $T_{x y}(x, 1 / 2)$ departs further from a parabola and breaks its symmetry as $R e \rightarrow 0$ with a fixed Mach number. Along the vertical centreline, the nonNewtonian nature of the LBM for appreciable Knudsen numbers is seen everywhere. Although the profiles are similar when $R e=10$ and $R e=0.5$, there is a significant departure form Newtonian behaviour when $R e=0.1$, where gradient terms that do not appear in the viscous stress are larger.

\section{Discussion}

Boundary conditions for the lattice Boltzmann equation are usually formulated as constraints on the incoming distribution functions but often have an entirely equivalent interpretation in terms of moment constraints $[34,35]$. Considering boundary conditions on the $\bar{f}_{i}$ (or $f_{i}$ in most standard LBMs) seems natural but it makes it difficult to guarantee the precise satisfaction of hydrodynamic conditions at boundaries, especially conditions more complex than no-slip walls, such as slip, stress or pressure conditions, for example. An alternative approach is to consider imposing constraints directly on the hydrodynamic moments, which can then be translated into the set of incoming $\bar{f}_{i}$. The "momentbased method" of Bennett [32] may be viewed as an extension and generalisation of previous work by Noble [29] and Wagner and Yeomans [31]. By considering linearly independent moments at a boundary one can impose three physically meaningful boundary conditions. This brings the opportunity to implement a wider variety of boundary conditions for the lattice Boltzmann equation. The original moment-based method imposed a Navier-Stokes condition on the tangential stress, $T_{x x}=\Pi_{x x}^{(0)}-\Pi_{x x}=0$. However, a more detailed inspection shows that the lattice Boltzmann equation includes a non-zero contribution to the stress at the Burnett level [12]. Boundary conditions imposed on the stress should incorporate this order $\mathcal{O}\left(\tau^{2}\right)$ term to be consistent with the stress in the equivalent PDE moment system. We have analysed the LBM stress field, highlighted its non-Newtonian behaviour, and derived consistent and quite general boundary conditions that can be imposed directly on the LBM moments. The conditions are complex when the boundary behaviour is complex, involving spatially-dependent velocities and derivatives, but they are translated into relatively simple conditions on the incoming $\bar{f}_{i}$ that are implemented locally and precisely.

The accuracy of the method has been assessed using the regularized lid-driven cavity flow [44]. The regularization removes the stress singularity in the upper corners of the cavity by setting the velocity of the upper boundary to $u_{x}(x)=16 x^{2}(1-x)^{2}$. The spatial gradient of the velocity influences the equilibrium and non-equilibrium parts of the momentum flux, $\Pi_{x x}$, and this should be respected by the boundary conditions. We have shown how this can be achieved locally within the consistent framework for the stress. While it is clear that the implementation is more complicated that bounce-back, for example, and thus computationally more expensive, the locality of the implementa- 
tion still allows us to exploit massively parallel modern computer architectures, leading to intensive and fast algorithms. Furthermore, the moment-based method is comparable to other commonly used lattice Boltzmann boundary treatments such as non-equilibrium bounce-back in terms of the number of operations required and recent work implementing the moment-based approach with CUDA and MPI parallelisations performs very well in terms of strong and weak scaling laws (95\% of the ideal strong scaling law) [59]. Numerically favourable values of the so-called "magic parameter" have been used to ensure stable simulations even on very coarse grids and the results are in excellent agreement with benchmark spectral data. Furthermore, a recently-published correction to the third-order non-Galilean invariant error in the momentum flux has been included and tested within our boundary conditions [10].

The LBM stress field has been computed and shown to depart significantly from the Navier-Stokes stress when the ratio $M a / \operatorname{Re} \propto K n \gg 0$. This is due to the contributions to the deviatoric stress at Burnett order. They do not vanish with mesh refinement under the acoustic scaling and are present with fixed Mach, Reynolds, and Knudsen numbers. The non-Newtonian stress is a feature of the LBM PDE moment system that may need to be considered in low Reynolds number flows. It should not be neglected in rarefied flow, although further analysis and research is needed to qualify and quantify its physical characteristics. If one is simulating the stress in Navier-Stokes flow at small Re with the LBM then a very small timestep may be needed to quash the Burnett contributions. In addition, it is common to use the non-equilibrium part of the moment flux, $\Pi_{\alpha \beta}^{(n e)}=$ $\Pi_{\alpha \beta}-\Pi_{\alpha \beta}^{(0)}$, to obtain the gradients of the velocity field locally in the LBM. This may be used for post-precessing or as part of the model (e.g shear-dependent viscosities in LBMs for generalised Newtonian fluids). When $\mathrm{Ma} / \mathrm{Re}$ is not vanishing small, additional and large contributions to $\Pi_{\alpha \beta}^{(n e)}$ may have a negative effect on the accuracy of the algorithm.

Like all lattice Boltzmann boundary implementations, the moment-based method has potential shortcomings: its current lack of geometric flexibility appears to be the most obvious. It does, however, have the attractive prospect of allowing for the exact satisfaction of complicated and sophisticated boundary conditions locally at grid points. It allows for a consistent stress condition to be imposed with relative ease and, as confirmed by the current assessment, this is achieved without sacrificing the second-order accuracy of the lattice Boltzmann equation.

\section{Acknowledgments}

The author would like to thank Professor Paul J. Dellar sincerely for his very helpful comments and advice. This research forms part of the actives of the UK Consortium on Mesoscopic Engineering Science (UKCOMES) [grant number EP/L00030X/1]. 


\section{Appendix A: Results with constant boundary velocity}

Here we briefly present results for the simpler, classical, lid-driven cavity flow with a constant upper boundary velocity $u_{x}(x, 1)=1$. Table 5 shows the maximum value of the stream function and its location with the moment method with $\Lambda=1 / 4$ and $\Lambda=1 / 6$ on successive meshes. Second-order convergence is observed. A comparison between our results and existing benchmark data is given in Tables 6 and 7. We can conclude that the moment based boundary conditions can predict very accurate results for this benchmark flow. It is particularly revealing to compare our results with the spectral solutions of Botella et al. [60] and the implicit cell-centred finite volume solutions of Sahin and Owens [61]. There is an excellent agreement with these data sets.

Table 5: Mesh dependence of the primary and lower streamfunctions computed with the moment method.

\begin{tabular}{||c||ccc|ccc||}
\hline \multicolumn{1}{||c||}{} & \multicolumn{3}{c|}{$\Lambda=1 / 4$} & \multicolumn{3}{c||}{$\Lambda=1 / 6$} \\
\hline$R e=100$ & $\psi_{P}$ & $x$ & $y$ & $\psi_{P}$ & $x$ & $y$ \\
$N=17$ & 0.10022 & 0.6250 & 0.7500 & 0.10121 & 0.6250 & 0.7500 \\
$N=33$ & 0.10207 & 0.6250 & 0.7500 & 0.10227 & 0.6250 & 0.7500 \\
$N=65$ & 0.10208 & 0.6250 & 0.7344 & 0.10281 & 0.6250 & 0.7344 \\
$N=129$ & 0.10307 & 0.6172 & 0.7422 & 0.10308 & 0.6172 & 0.7344 \\
$N=513$ & 0.10324 & 0.6172 & 0.7383 & 0.10324 & 0.6172 & 0.7383 \\
$R e=1000$ & 0.10332 & 0.6152 & 0.7383 & 0.10332 & 0.6152 & 0.7383 \\
$N=33$ & 0.11725 & 0.5313 & 0.5625 & 0.12038 & 0.5313 & 0.5625 \\
$N=65$ & 0.11895 & 0.5313 & 0.5625 & 0.11956 & 0.5313 & 0.5625 \\
$N=129$ & 0.11904 & 0.5313 & 0.5664 & 0.11914 & 0.5313 & 0.5625 \\
$N=257$ & 0.11891 & 0.5313 & 0.5664 & 0.11899 & 0.5313 & 0.5664 \\
$N=513$ & 0.11890 & 0.5313 & 0.5664 & 0.11897 & 0.5313 & 0.5664 \\
\hline$R e=5000$ & & & & & & \\
$N=129$ & 0.12245 & 0.5156 & 0.5391 & 0.12321 & 0.5156 & 0.5391 \\
$N=257$ & 0.12244 & 0.5156 & 0.5352 & 0.12260 & 0.5156 & 0.5352 \\
$N=513$ & 0.12239 & 0.5156 & 0.5352 & 0.12241 & 0.5156 & 0.5352 \\
\hline$R e=7500$ & & & & & & \\
$N=257$ & 0.12261 & 0.5117 & 0.5352 & 0.12287 & 0.5117 & 0.5313 \\
$N=513$ & 0.12258 & 0.5137 & 0.5313 & 0.12258 & 0.5137 & 0.5315 \\
\hline
\end{tabular}


Table 6: Comparison of the streamfunction computed with the moment method with other methods.

\begin{tabular}{|c|c|c|c|c|c|c|c|c|c|}
\hline & \multicolumn{3}{|c|}{ Primary } & \multicolumn{3}{|c|}{ Left } & \multicolumn{3}{|c|}{ right } \\
\hline $\operatorname{Re}=100$ & $\psi_{P}$ & $x$ & $y$ & $\psi_{L}$ & $x$ & $y$ & $\psi_{R}$ & $x$ & $y$ \\
\hline Present $\Lambda=1 / 4$ & 0.1032 & 0.6172 & 0.7383 & $-2.101 \times 10^{-6}$ & 0.0352 & 0.0352 & $-1.364 \times 10^{-5}$ & 0.9453 & 0.0664 \\
\hline Present $\Lambda=1 / 6$ & 0.1032 & 0.6172 & 0.7383 & $-2.101 \times 10^{-6}$ & 0.0352 & 0.0352 & $-1.364 \times 10^{-5}$ & 0.9453 & 0.0664 \\
\hline Luo et al. TRT [41] & 0.1035 & 0.6150 & 0.7378 & $-1.781 \times 10^{-6}$ & 0.0341 & 0.0341 & $-1.270 \times 10^{-5}$ & 0.9425 & 0.0614 \\
\hline Hou et al. [40] & 0.1030 & 0.6196 & 0.7373 & $-1.720 \times 10^{-6}$ & 0.0392 & 0.0353 & $-1.220 \times 10^{-5}$ & 0.9451 & 0.0627 \\
\hline Ghia et al. [62] & 0.1034 & 0.6188 & 0.7375 & $-1.940 \times 10^{-6}$ & 0.0375 & 0.0313 & $-1.140 \times 10^{-5}$ & 0.9375 & 0.0563 \\
\hline Sahin and Owens [61] & 0.1035 & 0.6189 & 0.7400 & $-1.793 \times 10^{-6}$ & 0.0332 & 0.0352 & $-1.266 \times 10^{-5}$ & 0.9424 & 0.0610 \\
\hline$R e=400$ & & & & & & & & & \\
\hline Present $\Lambda=1 / 4$ & 0.1139 & 0.5547 & 0.6055 & $-1.463 \times 10^{-5}$ & 0.0508 & 0.0469 & $-6.499 \times 10^{-4}$ & 0.8867 & 0.1250 \\
\hline Present $\Lambda=1 / 6$ & 0.1139 & 0.5547 & 0.6055 & $-1.463 \times 10^{-5}$ & 0.0508 & 0.0469 & $-6.503 \times 10^{-4}$ & 0.8867 & 0.1250 \\
\hline Luo et al. TRT [41] & 0.1138 & 0.5546 & 0.6053 & $-1.426 \times 10^{-5}$ & 0.0517 & 0.0478 & $-6.441 \times 10^{-4}$ & 0.8840 & 0.1218 \\
\hline Hou et al. [40] & 0.1121 & 0.5608 & 0.6078 & $-1.300 \times 10^{-5}$ & 0.0549 & 0.0510 & $-6.190 \times 10^{-4}$ & 0.8902 & 0.1255 \\
\hline Ghia et al. [62] & 0.1139 & 0.5547 & 0.6055 & $-1.420 \times 10^{-5}$ & 0.0508 & 0.0469 & $-6.420 \times 10^{-4}$ & 0.8906 & 0.1250 \\
\hline Sahin and Owens [61] & 0.1139 & 0.5536 & 0.6075 & $-1.427 \times 10^{-5}$ & 0.0508 & 0.0461 & $-6.404 \times 10^{-4}$ & 0.8835 & 0.1203 \\
\hline$R e=1000$ & & & & & & & & & \\
\hline Present $\Lambda=1 / 4$ & 0.1189 & 0.5313 & 0.5664 & $-2.337 \times 10^{-4}$ & 0.0820 & 0.0781 & $-1.730 \times 10^{-3}$ & 0.8672 & 0.1133 \\
\hline Present $\Lambda=1 / 6$ & 0.1190 & 0.5313 & 0.5664 & $-2.337 \times 10^{-4}$ & 0.0820 & 0.0781 & $-1.737 \times 10^{-3}$ & 0.8633 & 0.1133 \\
\hline Luo et al. TRT [41] & 0.1188 & 0.5312 & 0.5663 & $-2.321 \times 10^{-4}$ & 0.0828 & 0.0789 & $-1.730 \times 10^{-3}$ & 0.8358 & 0.1000 \\
\hline Hou et al. [40] & 0.1178 & 0.5333 & 0.5647 & $-2.220 \times 10^{-4}$ & 0.0902 & 0.0784 & $-1.690 \times 10^{-3}$ & 0.8667 & 0.1137 \\
\hline Ghia et al. [62] & 0.1179 & 0.5313 & 0.5625 & $-2.310 \times 10^{-4}$ & 0.0859 & 0.0781 & $-1.750 \times 10^{-3}$ & 0.8594 & 0.1094 \\
\hline Sahin and Owens [61] & 0.1188 & 0.5335 & 0.5639 & $-2.330 \times 10^{-4}$ & 0.0826 & 0.0776 & $-1.724 \times 10^{-3}$ & 0.8658 & 0.1119 \\
\hline Botella et al. [60] & 0.1189 & 0.4692 & 0.5652 & $-2.335 \times 10^{-4}$ & 0.9167 & 0.0781 & $-1.729 \times 10^{-3}$ & 0.1360 & 0.1118 \\
\hline$R e=5000$ & & & & & & & & & \\
\hline Present $\Lambda=1 / 4$ & 0.1224 & 0.5156 & 0.5313 & $-1.372 \times 10^{-3}$ & 0.0742 & 0.1367 & $-3.087 \times 10^{-3}$ & 0.8047 & 0.0742 \\
\hline Present $\Lambda=1 / 6$ & 0.1226 & 0.5156 & 0.5313 & $-1.375 \times 10^{-3}$ & 0.0742 & 0.1367 & $-3.081 \times 10^{-3}$ & 0.8047 & 0.0742 \\
\hline Hou et al. [40] & 0.1214 & 0.5176 & 0.5373 & $-1.350 \times 10^{-3}$ & 0.0784 & 0.1372 & $-3.030 \times 10^{-3}$ & 0.8078 & 0.0745 \\
\hline Ghia et al. [62] & 0.1190 & 0.5117 & 0.5352 & $-1.360 \times 10^{-3}$ & 0.0703 & 0.1367 & $-3.080 \times 10^{-3}$ & 0.8086 & 0.0742 \\
\hline Sahin and Owens [61] & 0.1221 & 0.5134 & 0.5376 & $-1.369 \times 10^{-3}$ & 0.0720 & 0.1382 & $-3.065 \times 10^{-3}$ & 0.8081 & 0.0741 \\
\hline$R e=7500$ & & & & & & & & & \\
\hline Present $\Lambda=1 / 4$ & 0.1226 & 0.5117 & 0.5352 & $-1.522 \times 10^{-3}$ & 0.0625 & 0.1523 & $-3.245 \times 10^{-3}$ & 0.7891 & 0.0664 \\
\hline Present $\Lambda=1 / 6$ & 0.1228 & 0.5117 & 0.5352 & $-1.525 \times 10^{-3}$ & 0.0625 & 0.1523 & $-3.238 \times 10^{-3}$ & 0.7891 & 0.0664 \\
\hline Hou et al. [40] & 0.1217 & 0.5176 & 0.5333 & $-1.510 \times 10^{-3}$ & 0.0706 & 0.1529 & $-3.200 \times 10^{-3}$ & 0.7922 & 0.0667 \\
\hline Ghia et al. [62] & 0.1200 & 0.5117 & 0.5322 & $-1.470 \times 10^{-3}$ & 0.0645 & 0.1367 & $-3.280 \times 10^{-3}$ & 0.7814 & 0.0625 \\
\hline Sahin and Owens [61] & 0.1223 & 0.5134 & 0.5376 & $-1.520 \times 10^{-3}$ & 0.0645 & 0.1525 & $-3.223 \times 10^{-3}$ & 0.7894 & 0.0642 \\
\hline
\end{tabular}

\section{References}

[1] G. M. McNamara, G. Zanetti, Use of the Boltzmann equation to simulate lattice gas automata, Phys. Rev. Lett. 61 (1988) 2332-2335.

[2] Y. H. Qian, D. d'Humières, P. Lallemand, Lattice BGK models for Navier-Stokes equation, Europhys. Lett. 17 (1992) 479-484.

[3] D. d'Humières, Generalized lattice Boltzmann equations, Prog. Aeronaut. Astronaut. 159 (1992) 450-458.

[4] X. He, L.-S. Luo, Theory of the lattice Boltzmann method: From the Boltzmann equation to 
Table 7: Computed value of the streamfunction in the upper secondary eddy.

\begin{tabular}{||l||ccc||}
\hline \multicolumn{1}{|c||}{} & \multicolumn{3}{c||}{ Upper } \\
\hline $\operatorname{Re}=5000$ & $\psi_{U}$ & $x$ & $y$ \\
Present $\Lambda=1 / 4$ & $-1.529 \times 10^{-3}$ & 0.0703 & 0.9102 \\
Present $\Lambda=1 / 6$ & $-1.504 \times 10^{-3}$ & 0.0644 & 0.9102 \\
Hou et al. [40] & $-1.400 \times 10^{-3}$ & 0.0667 & 0.9059 \\
Ghia et al. [62] & $-1.460 \times 10^{-3}$ & 0.0625 & 0.9102 \\
Sahin and Owens [61] & $-1.438 \times 10^{-3}$ & 0.0621 & 0.9108 \\
\hline$R e=7500$ & & & \\
Present $\Lambda=1 / 4$ & $-2.218 \times 10^{-3}$ & 0.0664 & 0.9102 \\
Present $\Lambda=1 / 6$ & $-2.209 \times 10^{-3}$ & 0.0664 & 0.9102 \\
Hou et al. [40] & $-2.060 \times 10^{-3}$ & 0.0706 & 0.9098 \\
Ghia et al. [62] & $-2.050 \times 10^{-3}$ & 0.0664 & 0.9141 \\
Sahin and Owens [61] & $-2.120 \times 10^{-3}$ & 0.0670 & 0.9108 \\
\hline
\end{tabular}

the lattice Boltzmann equation, Phys. Rev. E 56 (1997) 6811.

[5] C. Obrecht, F. Kuznik, B. Tourancheau, J.-J. Roux, Scalable lattice Boltzmann solvers for CUDA GPU clusters, Parallel Computing 39 (2013) 259-270.

[6] M. Junk, Z. Yang, Asymptotic analysis of lattice Boltzmann boundary condition, J. Stat. Phys. 121 (2005) 3-35.

[7] X. He, Q. Zou, L.-S. Luo, M. Dembo, Analytic solutions of simple flows and analysis of nonslip boundary conditions for the lattice Boltzmann BGK model, J. Stat. Phys. 87 (1997) $115-136$.

[8] M. Junk, A. Klar, L.-S. Luo, Asymptotic analysis of the lattice Boltzmann equation, J. Comp. Phys. 210 (2005) 676-704.

[9] G. Hazi, P. Kavran, On the cubic velocity deviations in lattice Boltzmann methods, J. Phys. A 39 (2006) 3127-3136.

[10] P. J. Dellar, Lattice Boltzmann algorithms without cubic defects in Galilean invariance on standard lattices, J. Comput. Phys. 259 (2014) 270-283.

[11] M. Geier, M. Schönherr, A. Pasquaili, M. Krafczyk, The cumulant lattice Boltzmann equation in three dimensions: Theory and validation, Comput. Math. Appl. 70 (2015) 507-547.

[12] P. J. Dellar, Lattice Boltzmann formulation for linear viscoelastic fluids using an abstract second stress, SIAM J. Sci. Comput. 36 (2014) A2507-A2532.

[13] C. Cercignani, Kinetic theory with "bounce-back" boundary conditions, Transport Theor. Stat. 18 (1989) 125-131.

[14] A. J. C. Ladd, Numerical simulations of particulate suspensions via a discretized Boltzmann equation. Part 1. Theoretical foundation, J. Fluid Mech. 271 (1994) 285-309.

[15] I. Ginzbourg, M. P. Adler, Boundary flow condition analysis for the three-dimensional lattice Boltzmann model, J. Phys. II 4 (1994) 191-214.

[16] D. d'Humières, I. Ginzburg, Viscosity independent numerical errors for Lattice Boltzmann models: From recurrence equations to "magic" collision numbers, Comput. Math. Appl. 58 (2009) 823-840. 
[17] I. Ginzburg, D. d'Humières, A. Kuzmin, The role of kinetic parameter in the stability of two-relaxation-time advection-diffusion lattice Boltzmann schemes, Comp. Math. Appl. 139 (2010) 1090-1143.

[18] D. J. Holdych, D. R. Noble, J. G. Georgiadis, Truncation error analysis of lattice Boltzmann methods, J. Comp. Phys. 193 (2004) 595-619.

[19] A. Kuzmin, I. Ginzburg, M. M. Mohamad, Optimal stability of advection-diffusion lattice Boltzmann models with two relaxation times for positive/negative equilibrium, J. Stat. Phys. 139 (2010) 1090-1143.

[20] I. Ginzburg, F. Verhaeghe, D. d'Humières, Study of simple hydrodynamic solutions with the two-relaxation-times lattice Boltzmann scheme, Commun. Comput. Phys. 3 (2008) 519-581.

[21] J. C. Maxwell, On stresses in rarified gases arising from inequalities of temperature, Phil. Trans. Royal Soc. 170 (1879) 231-256.

[22] J. E. Broadwell, Study of rarefied shear flow by the discrete velocity method, J. Fluid Mech. 19 (1963) 401-414.

[23] R. Gatignol, Kinetic theory boundary conditions for discrete velocity gases, Phys. Fluids 20 (1977) 2022-2030.

[24] S. Ansumali, I. Karlin, Kinetic boundary conditions in the lattice Boltzmann method, Phys. Rev. E 66 (2002) 026311.

[25] S. H. Kim, H. Pitsch, I. D. Boyd, Accuracy of higher-order lattice Boltzmann methods for microscale flows with finite Knudsen numbers, J. Comp. Phys. 227 (2008) 8655-8671.

[26] P. Brookes, Lattice Boltzmann in the finite knudsen number flow regime, Master's thesis, University of Melbourne (2006).

[27] W. Yudistiawan, S. Ansumali, I. Karlin, Hydrodynamics beyond Navier-Stokes: The slip flow model, Phy. Rev. E 78 (2008) 016705.

[28] F. Verhaeghe, L.-S. Luo, Lattice Boltzmann modeling of microchannel flow in slip flow regime, J. Comp. Phys. 228 (2009) 147-157.

[29] D. R. Noble, S. Chen, J. G. Georgiadis, R. O. Buckius, A consistent hydrodynamic boundary condition for the lattice Boltzmann method, Phys. Fluids 7 (1995) 203-209.

[30] A. Frisch, B. Hasslacher, Y. Pomeau, Lattice-gas automata for the Navier-Stokes equation, Phys. Rev. Lett. 56 (1986) 1505-1508.

[31] A. J. Wagner, J. M. Yeomans, Phase separation under shear in two-dimensional binary fluids, Phys. Rev. E 59 (1999) 4366-4373.

[32] S. Bennett, A lattice Boltzmann model for diffusion of binary gas mixtures, Ph.D. thesis, University of Cambridge (2010).

[33] S. Mohammed, D. Graham, T. Reis, Assessing moment-based boundary conditions for the lattice Boltzmann equation: a study of dipole-wall collisions, Comput, Fluids. 176 (2018) 79-96.

[34] S. Bennett, P. Asinari, P. Dellar, A lattice Boltzmann model for diffusion of binary gas mixtures that includes diffusion slip, Int. J. Num. Meth. Fluids 69 (2012) 171-189.

[35] T. Reis, P. J. Dellar, Lattice Boltzmann simulations of pressure-driven flows in microchannels using Navier-Maxwell slip boundary conditions, Phys. Fluids 24 (2012) 112001.

[36] Z. B. Sinnah, D. Graham, T. Reis, Lattice Boltzmann modelling of pulsatile flow using moment boundary conditions, in: Proceedings of the 6th European Conference on Computational Mechanics and 7th European Conference on Computational Fluids Dynamics, 2012.

[37] A. Hantsch, T. Reis, U. Gross, Moment method boundary conditions for multiphase lattice Boltzmann simulations with partially-wetted walls, J. Comput. Multiphase Flows 7 (2015) 1-14. 
[38] R. Allen, T. Reis, Moment-based boundary conditions for lattice Boltzmann simulations of natural convection in cavities, Prog. Comput. Fluid. Dy. 16 (2016) 216-231.

[39] S. Mohammed, T. Reis, Using the lid-driven cavity flow to validate moment-based boundary conditions for the lattice Boltzmann equation, Arch. Mech. Eng. 64 (1) (2017) 57-74.

[40] S. Hou, Y. Zu, S. Chen, G. Doolen, A. C. Cogley, Simulation of cavity flow by the lattice Boltzmann method, J. Comp. Phys. 118 (1995) 329-347.

[41] L.-S. Luo, W. Liao, X. Chen, Y. Peng, W. Zhang, Numerics of the lattice Boltzmann method: Effects of collision models on the lattice Boltzmann simulations, Phys. Rev. E 83 (2011) 056710 .

[42] A. N. Gorban, D. J. Packwood, Enhancement of the stability of lattice Boltzmann methods by dissipation control, Physica A 414 (2012) 285-299.

[43] R. A. Brownlee, J. Levesley, D. J. Packwood, A. N. Gorban, Add-ons for lattice Boltzmann methods: Regularizationm, filtering and limiters, in: M. Ehrhardt (Ed.), Novel trends in lattice Boltzmann methods - reactive flows, physiochemical transport and fluid-structure interaction, Bentham Science Publishers, 2012, pp. 29-50.

[44] J. Shen, Hopf bifurcation of the unsteady regularized driven cavity flow, J. Comp. Phys. 95 (1991) 228-245.

[45] X. Shan, X. He, Discretization of the velocity space in the solution of the Boltzmann equation, Phys. Rev. Lett 80 (1998) 65-68.

[46] T. I. Gombosi, Gaskinetic Theory, Cambridge Universty Press, 1994.

[47] S. Chapman, T. C. Cowling, The Mathematical Theory of Non-uniform Gases, Cambridge University Press, 1970.

[48] C. Cercignani, The Boltzmann equation and its applications, 1st Edition, Springer-Verlag, New York, 1988.

[49] Y. Qian, S. Orszag, Non-linear correction to Navier-Stokes equation derived from lattice BGK models, Europhys. Lett. 21 (1993) 255-259.

[50] X. He, X. Shan, G. Doolen, Discrete Boltzmann equation model for nonideal gases, Phys. Rev. E 57 (1998) R13.

[51] P. J. Dellar, Incompressible limits of lattice Boltzmann equations using multiple relaxation times, J. Comp. Phys. 190 (2003) 351-370.

[52] P. Lallemand, L.-S. Luo, Theory of the lattice Boltzmann method: Dispersion, dissipation, isotropy, Galilean invariance, and stability, Phys. Rev. E 61 (2000) 6546-6562.

[53] J. Latt, B. Chopard, Lattice Boltzmann method with regularized precollision distribution functions, Math. Comp. Sim 72 (2006) 165-168.

[54] I. Ginzburg, Equilibrium-type and link-type lattice Boltzmann models for generic advection and anisotropic-dispersion equation, Adv. Water Resour. 28 (2005) 1171-1195.

[55] E. Ickenberry, C. Truesdell, On the pressures and the flux of energy in a gas according to Maxwell's kinetic theory, I, J. Rational Mech. Anal. 1 (1956) 1-54.

[56] C. Truesdell, On the pressures and the flux of energy in a gas according to Maxwell's kinetic theory, II, J. Rational Mech. Anal. 1 (1956) 55-127.

[57] H. Barnes, J. Hutton, K. Walters, An Introduction to Rheology, 1st Edition, Elsevier, Amsterdam, The Netherlands, 1989.

[58] W. H. Press, S. A. Teukolsky, W. T. Vetterling, B. P. Flannery, Numerical Recipes in FORTRAN; The Art of Scientific Computing, 2nd Edition, Cambridge University Press, New York, NY, USA, 1993.

[59] I. Krastins, Parallel lattice Boltzmann method for convection in dendritic solidification, Ph.D. thesis, University of Greenwich (2018). 
[60] O. Botella, R. Peyret, Benchmark spectral results on the lid-driven cavity flow, Comput. Fluids 27 (1998) 421-433.

[61] M. Sahin, R. G. Owens, A novel fully implicit finite volume method applied to the lid-driven cavity problem - Part 1: High Reynolds number flow calculations, Int. J. Numer. Meth. Fluids 42 (2003) 57-77.

[62] U. Ghia, K. N. Ghia, C. T. Shin, High-Re solutions for incompressible flow using the NavierStokes equations and a multigrid method, J. Comp. Phys. 48 (1982) 387-411. 